\title{
ZERO-SUM MARKOV GAMES WITH IMPULSE CONTROLS*
}

\author{
ARNAB BASU† AND ŁUKASZ STETTNER
}

\begin{abstract}
In this paper we consider a zero-sum Markov stopping game on a general state space with impulse strategies and infinite time horizon discounted payoff where the state dynamics is a weak Feller-Markov process. One of the key contributions is our analysis of this problem based on "shifted" strategies, thereby proving that the original game can be practically restricted to a sequence of Dynkin's stopping games without affecting the optimalty of the saddle-point equilibria and hence completely solving some open problems in the existing literature. Under two quite general (weak) assumptions, we show the existence of the value of the game and the form of saddle-point (optimal) equilibria in the set of shifted strategies. Moreover, our methodology is different from the previous techniques used in the existing literature and is based on purely probabilistic arguments. In the process, we establish an interesting property of the underlying Feller-Markov process and the impossibility of infinite number of impulses in finite time under saddle-point strategies which is crucial for the verification result of the corresponding Isaacs-Bellman equations.
\end{abstract}

Key words. Feller-Markov processes, stopping times, zero-sum games, Isaacs-Bellman equations, Dynkin's stopping game, impulse controls, saddle-point strategies, discounted cost

AMS subject classifications. 93E20, 60J25, 60J05, 93C55

DOI. $10.1137 / 18 \mathrm{M} 1229365$

1. Introduction. The subject of zero-sum stochastic games with optimal stopping was initiated by Dynkin in [7]. The results therein were extended to the continuous time case by Krylov in [14], [15]. Such games were later studied by Bensoussan and Friedman in [2] for diffusion processes using variational inequalities and by Bismut [3] using convex-analytic methods. These so-called Dynkin games were studied in [22] in a general Markov setting with infinite horizon and fixed discount rate under reasonably general assumptions (conditions) as introduced by Robin in [21] for Markov stopping problems. Cvitanic and Karatzas [5] studied such games using a backward stochastic differential equation (BSDE) approach. More recently, the analysis of the existence of value of such games in continuous time and corresponding mixed optimal strategies were studied in [8], [16], [26], and the references therein.

Besides being important from a game theory point of view, such stopping games with impulsive strategies have quite important applications in the mathematics of the finance-like pricing of American options and game (Israeli) options (see, e.g., [10], [11], and [13] and references therein) and pricing of Israeli swing options with multiple exercises of derivatives as in [12] (see also [4] for other applications).

Zero-sum impulse games were first considered in [22], where fixed execution delay for the impulses was considered (see also [24]). In this paper we consider so-called decision lag. The assumption (A1) imposed in this paper (see the next section) states that after an impulse the next one is allowed only after some lag depending on the values of the process before and after this shift. As we have shown in Example 1

${ }^{*}$ Received by the editors November 28, 2018; accepted for publication (in revised form) December 4, 2019; published electronically February 27, 2020.

https://doi.org/10.1137/18M1229365

Funding: The second author gratefully acknowledges research support from the National Science Center of Poland by grant UMO-2016/23/B/ST1/00479.

$\dagger$ Department of Industrial Engineering, Bilkent University, Ankara 06-800, Turkey (arnab@ bilkent.edu.tr).

${ }^{\ddagger}$ Institute of Mathematics, Polish Academy of Sciences (IMPAN), Warsaw, 00-656 Poland (stettner@impan.pl). 
this kind of assumption is necessary to avoid an infinite number of shifts at the same time point. Looking for minimal assumptions we introduce a weaker assumption (A2) which imposes decision lag after the impulse of the maximizer assuming that the immediate previous impulse was made by the minimizer. A related assumption was introduced in [4] to guarantee a finite number of impulses in a finite interval and it was used therein to study impulse games with diffusion process and viscosity solutions to corresponding quasivariational inequalities.

In this paper we have a more general state process and use purely probabilistic arguments which are different from the previous methodology used in the existing literature. In particular, herein we are interested in a Markov game where the payoffs are functionals of the current values of a given Markov process $\{X(s)\}_{s \geq 0}$. The values of the Dynkin games exist in a general setting when these functionals are only right continuous processes (see, e.g., [17], [25], and also [16], [26] for games with randomized stopping). In his paper [22], the second author addressed this problem under so-called fixed execution delay, i.e., when shift at time $\tau$ was decided to be $\xi$ but it was executed at time $\tau+h$ to $\xi$ (which was $F_{\tau}$ measurable), where $h>0$ is fixed beforehand. In this work, we consider a generalization of that zero-sum stopping game as in [22] and show the existence of the value of such a game and the form of saddle-point (optimal) strategies under two quite general (weaker) assumptions (A1) and (A2) as mentioned above and described in the next section. As is shown, this game can be practically restricted to a sequence of Dynkin's stopping games. We also provide a counterexample (Example 1) to prove their necessity for the value function to be unique. As is shown in this counterexample the game with impulses makes sense only when there is some kind of assumption which enables us to restrict an infinite number of immediate impulses. Such a condition assumed in [22] has fixed execution delay. In this paper we first have decision lag (after a shift without any execution delay the next shift is allowed only after some lag depending on the state of the process before and after the shift) required for both players (Assumption (A1)). By relaxing this assumption we introduce (A2), which seems to be the minimal assumption under which we still have the value of the game. This assumption says that after the impulse of the minimizer if we have the impulse of the maximizer, then thereafter a decision lag follows, i.e., for the next shift we have to wait $h$ units of time which depend on the value of the process before and after the shift (of the maximizer).

There are two recent papers [1] and [9] in which a zero sum stochastic differential game was considered. In [1] one player used impulse controls while the other used continuous time stochastic control. In [9] both players used impulse controls and under certain strong set of assumptions it was shown that the value function of the game was a viscosity solution to a suitable Hamilton-Jacobi-Bellman-Isaacs equation, but the authors were not able to find saddle-point strategies. In this paper we solve this open problem completely under a very general set of assumptions and establish the corresponding saddle-point (optimal) strategies, thereby generalizing in particular [9].

This paper is organized as follows. The next section describes the overall problem structure and formulation as well as the generic assumptions under which the results of this paper shall be proved. The Markov version of the continuous-time Dynkin game is studied in section 3, where we prove the existence of value and saddle-point (optimal) strategies for such a game. Section 4 describes the dynamic programming formulation of our game and states the two main theorems of this paper, namely, the existence of unique continuous bounded solutions to the Isaacs-Bellman equation (Theorem 4.1) and the verification result that this solution is indeed the value of our game (Theorem 4.2). This section also aptly provides a counterexample (Example 1) to show that 
these weak assumptions made in section 2 are necessary for the uniqueness of the value function. Restricting to the so-called "shifted" strategies of the players (see the next section for a description), section 5 proves Theorem 4.1 under the assumption (A1) of section 2 whereas section 6 does so under the assumption (A2) of section 2 . In this section 6 we also, under the assumption (A2), propose and prove several important results, namely, that the successive impulses by either player can occur only with very low probability (Lemmas 6.5 and 6.6) and the crucial fact (Proposition 6.7) that an infinite number of impulses cannot happen in finite time. To prove these results we study certain properties of Feller-Markov processes by using Lemmas 5.1 and 6.3 as well as by proposing and proving Proposition 6.4, which can be of independent interest. Under either of the assumptions (A1) or (A2), section 7 proves Theorem 4.2 in full generality, i.e., the value of the game does not change if the players use the most general (history-dependent) class of admissible impulse strategies instead of only shifted strategies. In fact, we prove the existence of a saddle-point equilibrium within the set of shifted strategies. Finally, we conclude with a few comments and proposals for future directions of work in section 8 .

2. Problem formulation. This section describes our general game framework as well the (weak) assumptions under which our results shall hold. Given a locally compact separable metric space $E$ endowed with a metric $\rho$, let $\tilde{\Omega} \stackrel{\text { def }}{=} \mathcal{D}([0, \infty) ; E)$ be the space of cadlag functions from $\mathbb{R}_{+}$to $E$ and $\tilde{\mathcal{F}},\left\{\tilde{\mathcal{F}}_{t}\right\}$ are universally completed $\sigma$-fields of the canonical space $\tilde{\Omega}$. We consider the state process $\left\{X_{s}\right\}_{s \geq 0}$ to be a standard Feller-Markov process $\left(X_{s}(\omega)=\omega(s), \omega \in \tilde{\Omega}\right)$ defined on a probability space $\left(\tilde{\Omega}, \tilde{\mathcal{F}},\left\{\tilde{\mathcal{F}}_{t}\right\}, P\right)$ taking values in $E$ with shift operator $\theta_{t}$ and conditional law $P_{x}$ when it starts from $x$. For any space $S$, we denote by $C(S)$ the space of bounded, continuous, and real-valued functions on $S$. Let $f \in C(E)$ and $c, d \in C(E \times E)$ be given. We shall assume that the transition operator of the Markov process transforms the space $C_{0}(E)$ of continuous functions vanishing at infinity into itself. Let $U_{1}, U_{2}$ be compact subsets of $E$. We define $B(U, R) \equiv\left\{x \in E: \inf _{y \in U} \rho(x, y) \leq R\right\}$ for given $R>0$ and $U$ a compact subset of $E$. In particular, for any $x \in E$ if $U=\{x\}$, then $B(U, R)$ denotes the closed ball with radius $R$ and center $x$. Let $\left\{\tau_{i}\right\}_{i=1,2, \ldots},\left\{\sigma_{i}\right\}_{i=1,2, \ldots}$ be stopping times and $\left\{\xi_{i}\right\}_{i=1,2, \ldots},\left\{\zeta_{i}\right\}_{i=1,2, \ldots}$ (resp.) be random variables measurable with respect to available information till $\tau_{i}, \sigma_{i}$ (resp.) taking values in (resp.) $U_{1}, U_{2}$. Let $\tau_{i} \wedge \sigma_{i}:=$ $\rho_{i}$ and assume that $\tau_{i+1} \wedge \sigma_{i+1} \geq \rho_{i}$, for $i=1,2, \ldots$ Note that here we use somewhat of a barbarism of notation between the impulse moment and the metric on $E$ and the usage should be clear from the context. We consider a zero-sum game between two players I and II, where player I chooses strategy $V_{1} \stackrel{\text { def }}{=}\left\{\tau_{1}, \xi_{1} ; \tau_{2}, \xi_{2} ; \ldots\right\}$ to maximize his payoff (described below) and player II chooses strategy $V_{2} \stackrel{\text { def }}{=}\left\{\sigma_{1}, \zeta_{1} ; \sigma_{2}, \zeta_{2} ; \ldots\right\}$ to minimize the same. At time $\rho_{i}$ the process is shifted to $\xi_{i}$ when $\tau_{i} \leq \sigma_{i}$ or to $\zeta_{i}$ when $\sigma_{i}<\tau_{i}$. The infinite-horizon discounted payoff under strategy-tuple $\left(V_{1}, V_{2}\right)$ starting at time 0 from the point $x \in E$ is defined as

$$
\begin{aligned}
& \mathcal{J}^{V_{1}, V_{2}}(x) \stackrel{\text { def }}{=} E_{x}^{V_{1}, V_{2}}\left[\int_{0}^{\infty} e^{-\alpha s} f\left(Y_{s}\right) d s\right. \\
& \left.\quad+\sum_{i=1}^{\infty} e^{-\alpha\left(\tau_{i} \wedge \sigma_{i}\right)}\left(\mathbf{1}_{\left\{\tau_{i} \leq \sigma_{i}\right\}} c\left(X_{\tau_{i}}^{-}, \xi_{i}\right)+\mathbf{1}_{\left\{\sigma_{i}<\tau_{i}\right\}} d\left(X_{\sigma_{i}}^{-}, \zeta_{i}\right)\right)\right],
\end{aligned}
$$

where $\alpha>0$ is the discount factor, $X^{-}$denotes the value of the controlled process $\left\{X_{s}\right\}_{s \geq 0}$ just before the corresponding impulsive shift is made and to avoid infin- 
itely many shifts for gain by any player $c(\cdot, \cdot)<0$ and $d(\cdot, \cdot)>0$ with the natural assumptions that for $x \in E, \xi_{1}, \xi_{2} \in U_{1}$ and $\zeta_{1}, \zeta_{2} \in U_{2}$

$$
c\left(x, \xi_{1}\right)>c\left(x, \xi_{2}\right)+c\left(\xi_{2}, \xi_{1}\right)
$$

and

$$
d\left(x, \zeta_{1}\right)<d\left(x, \zeta_{2}\right)+d\left(\zeta_{2}, \zeta_{1}\right)
$$

The description of the process $Y$ follows soon. The interpretation is that player I (resp., player II) chooses a random time $\tau_{i}$ (resp., $\sigma_{i}$ ) and shifts the process from $X_{\tau_{i}} \in$ $E$ (resp., $X_{\sigma_{i}} \in E$ ) to a point $\xi_{i} \in U_{1}$ (resp., $\zeta_{i} \in U_{2}$ ), thereby incurring a negative payoff $c\left(X_{\tau_{i}}, \xi_{i}\right)$ (resp., positive payoff $\left.d\left(X_{\sigma_{i}}, \zeta_{i}\right)\right)$ and this goes on ad infinitum. There is a running payoff denoted by a bounded function $f(\cdot)$ which accumulates over the entire time horizon.

To describe the evolution of the controlled Markov process $Y$ we have to construct a suitable probability space following [18] and [21] (see in particular Annexes 1 and 2 therein). Denoting by $N$ positive integers define the Cartesian product $\Omega:=\tilde{\Omega}^{N}$ endowed with product $\sigma$-field $\mathcal{F}:=\tilde{\mathcal{F}}^{N}$, and analogously $\mathcal{F}^{i}:=\tilde{\mathcal{F}}^{i}$ and $\mathcal{F}_{t}:=\left\{\tilde{\mathcal{F}}_{t}^{N}\right\}$, $\mathcal{F}_{t}^{i}:=\left\{\tilde{\mathcal{F}}_{t}^{i}\right\}$, for $i=1,2, \ldots$, where to simplify notation we assume that $\mathcal{F}\left(\mathcal{F}^{i}\right)$ and $\mathcal{F}_{t}\left(\mathcal{F}_{t}^{i}\right)$ are universally completed $\sigma$-fields of $\tilde{\mathcal{F}}^{N}\left(\tilde{\mathcal{F}}^{i}\right)$ or $\left\{\tilde{\mathcal{F}}_{t}^{N}\right\}\left(\left\{\tilde{\mathcal{F}}_{t}^{i}\right\}\right)$, respectively. Also define $\omega:=\left(\omega_{1}, \omega_{2}, \ldots\right)$ and $[\omega]_{i}:=\left(\omega_{1}, \ldots, \omega_{i}\right)$ and let $\tau_{i}(\omega)=\tau_{i}\left([\omega]_{i}\right)$ and $\sigma_{i}(\omega)=\sigma_{i}\left([\omega]_{i}\right)$ be $\mathcal{F}_{t}^{i}$-stopping times. Then the controlled process $\left\{Y_{s}\right\}_{s \geq 0}$ can be described as follows: $Y_{s}(\omega):=X_{s}^{1}\left([\omega]_{1}\right):=\omega_{1}(s)$ for $s<\rho_{1}, Y_{s}(\omega):=X_{s}^{2}\left([\omega]_{2}\right):=\omega_{2}(s)$ for $\rho_{1}\left(\omega_{1}\right) \leq s<\rho_{2}\left(\omega_{1}, \omega_{2}\right)$, and $Y_{s}(\omega):=X_{s}^{i}\left([\omega]_{i}\right):=\omega_{i}(s)$ for $\rho_{i-1}\left([\omega]_{i-1}\right) \leq s<$ $\rho_{i}\left([\omega]_{i}\right)$, with $i=3,4, \ldots$. Similarly $\xi_{i}(\omega):=\xi_{i}\left([\omega]_{i}\right)\left(\zeta_{i}(\omega):=\zeta_{i}\left([\omega]_{i}\right)\right)$ are $\mathcal{F}_{\tau_{i}}^{i}$ (resp., $\left.\mathcal{F}_{\sigma_{i}}^{i}\right)$ measurable $U_{1}\left(\right.$ resp., $\left.U_{2}\right)$-valued random variables. Let $\mathcal{G}_{\rho_{i}}^{i}:=\sigma\left\{\mathcal{F}_{\rho_{i}-}^{i+1}, \mathcal{F}_{\rho_{i}}^{i} \otimes\right.$ $\{\emptyset, \tilde{\Omega}\}\}$ for $i=1,2, \ldots$ For given control strategies $V_{1}$ and $V_{2}$ there exists (see [18] and [21] for the detailed construction) a probability measure $P_{x}^{V_{1}, V_{2}}$ such that

$$
P_{x}^{V_{1}, V_{2}}\left(\theta_{\rho_{i}}^{-1} A \mid \mathcal{G}_{\rho_{i}}^{i}\right)=\delta_{X_{\rho_{i}}^{1}} \otimes \cdots \otimes \delta_{X_{\rho_{i}}^{i}} \otimes P_{X_{\rho_{i}}^{i+1}}\{A\}
$$

where $A \in \mathcal{F}$ and $\theta:=\left\{\theta_{s}\right\}_{s \geq 0}$ is a shift operator on $\Omega$ defined as $\theta_{s}: \Omega \ni \omega \mapsto$ $\omega(s+\cdot) \in \Omega$, i.e., $X_{s}(\omega)=X_{0}\left(\theta_{s}(\omega)\right)$. Consequently the cost functional (2.1) can be written as follows:

$$
\begin{aligned}
& \mathcal{J}^{V_{1}, V_{2}}(x) \stackrel{\text { def }}{=} E_{x}^{V_{1}, V_{2}}\left[\int_{0}^{\infty} e^{-\alpha s} f\left(Y_{s}\right) d s\right. \\
& \left.+\sum_{i=1}^{\infty} e^{-\alpha\left(\tau_{i} \wedge \sigma_{i}\right)}\left(\mathbf{1}_{\left\{\tau_{i} \leq \sigma_{i}\right\}} c\left(X_{\tau_{i}}^{i}, \xi_{i}\right)+\mathbf{1}_{\left\{\sigma_{i}<\tau_{i}\right\}} d\left(X_{\sigma_{i}}^{i}, \zeta_{i}\right)\right)\right] .
\end{aligned}
$$

We have therefore an impulse game such that the players first choose stopping times $\tau_{i}$ and $\sigma_{i}$ and their suggested shifts of the state process to $\xi_{i}$ or to $\zeta_{i}$, respectively, and then at $\rho_{i}=\tau_{i} \wedge \sigma_{i}$ the state process is shifted to $\xi_{i}$ when $\tau_{i} \leq \sigma_{i}$ with cost $c\left(X_{\tau_{i}}^{i}, \xi_{i}\right)$ while when $\sigma_{i}<\tau_{i}$ the state process is shifted to $\zeta_{i}$ with cost $d\left(X_{\sigma_{i}}^{i}, \zeta_{i}\right)$. In this description the first player has a priority in the sense that when both players decide to shift the process simultaneously, it is shifted according to the first player's choice. After each shift of the process the game starts afresh and the players choose their next stopping times and impulses. With such a game we can associated upper 
$\bar{v}$ and lower values $\underline{v}$ defined as follows:

$$
\begin{aligned}
& \bar{v}(x) \stackrel{\text { def }}{=} \inf _{V_{2} \in \mathcal{V}_{2}} \sup _{V_{1} \in \mathcal{V}_{1}} \mathcal{J}^{V_{1}, V_{2}}(x), \\
& \underline{v}(x) \stackrel{\text { def }}{=} \sup _{V_{1} \in \mathcal{V}_{1}} \inf _{V_{2} \in \mathcal{V}_{2}} \mathcal{J}^{V_{1}, V_{2}}(x)
\end{aligned}
$$

with the meaning that in the case of the upper value of the game the first player knows the stopping time and impulse of the second player while in the case of the lower value of the game the second player knows the stopping time and impulse of the first player. In Definition (2.6) above, $\mathcal{V}_{1}$ (resp., $\mathcal{V}_{2}$ ) denote the set of general admissible (dependent on the whole histories) strategies of player I (resp., player II).

To avoid an infinite number of shifts (impulses) at the same time we have to make one of the following assumptions by introducing a continuous bounded strictly positive function $h$ :

(A1) There is a decision lag: if an impulse to $\xi_{i} \in U_{1}$ or to $\zeta_{i} \in U_{2}$ is made at time $\rho_{i}$, then the next stopping times $\tau_{i+1}$ and $\sigma_{i+1}$ should be greater or equal to $\hat{\rho}_{i}:=\rho_{i}+h\left(X_{\rho_{i}}^{i}, \xi_{i}\right)$ or $\hat{\rho}_{i}:=\rho_{i}+h\left(X_{\rho_{i}}^{i}, \zeta_{i}\right)$ depending on whether impulse to $\xi_{i}$ or to $\zeta_{i}$ was executed.

(A2) After each shift of the minimizer followed by the maximizer there is a decision lag: if at time $\sigma_{i}$ the shift of the minimizer to $\zeta_{i} \in U_{2}$ is made and the next stopping time is $\tau_{i+1}$ chosen by the maximizer with shift $\xi_{i+1}$, then the next stopping time should not be smaller than $\tau_{i+1}+h\left(X_{\tau_{i+1}}^{i+1}, \xi_{i+1}\right)$.

Notice that although decision lag is assumed to be strictly positive we do not assume that it is bounded away from 0 , so that, when $x$ is large, $h(x, z), z \in U_{1}, U_{2}$ may be very small, which corresponds to an almost immediate correction of the state process when its value is far away from the compact sets $U_{1}, U_{2}$.

In our construction of strategies we shall restrict ourselves first to shifted strategies, i.e., we assume that for a given $\hat{\rho}_{i-1}, i=1,2, \ldots$ with $\hat{\rho}_{0}=0$ the strategies of the players are of the form $\tau_{i}=\hat{\rho}_{i-1}+\tau^{i} \circ \theta_{\hat{\rho}_{i-1}}$ and $\sigma_{i}=\hat{\rho}_{i-1}+\sigma^{i} \circ \theta_{\hat{\rho}_{i-1}}$, where $\tau^{i}$ and $\sigma^{i}$ are stopping times and $\xi_{i}=\xi^{i} \theta_{\hat{\rho}_{i-1}}, \zeta_{i}=\zeta^{i} \theta_{\hat{\rho}_{i-1}}$ with $\xi^{i}$ (resp., $\zeta^{i}$ ) being $U_{1}$ (resp., $U_{2}$ )-valued random variables adapted to the $\sigma$-fields generated by $X_{\tau_{i}}^{i}$ (resp., $X_{\sigma_{i}}^{i}$ ), implying they are not dependent on the whole histories $\tilde{\mathcal{F}}_{\tau_{i}}$ or $\tilde{\mathcal{F}}_{\sigma_{i}}$, respectively, and where $\hat{\rho}_{i}=\rho_{i}$ whenever there is no decision lag.

The purpose of this paper is to show the existence of the value of such a zero-sum game, i.e., $\bar{v}(x)=\underline{v}(x)$ for each $x \in E$, and to determine corresponding saddle-point strategies. Consequently the game can be restricted to the sequence of Dynkin's stopping games. For this purpose we are going to show the existence and regularity of solutions of suitable Isaacs-Bellman equations. We shall restrict ourselves first to shifted strategies and then using suitable verification results we shall show that the use of general admissible impulse strategies (dependent on the whole histories) does not change the value of the game and that there is a saddle-point (optimal) equilibrium in the set of shifted strategies.

3. Dynkin's game revisited. In this section we shall study a Markov version of the continuous time Dynkin's stopping game. There are two players choosing stopping times $\tau$ and $\sigma$ as their strategies. The functional is then of the form

$$
\begin{gathered}
I_{x}(\tau, \sigma):=E_{x}\left[\int_{0}^{\tau \wedge \sigma} e^{-\alpha s} f\left(X_{s}\right) d s+\mathbf{1}_{\{\tau \leq \sigma\}} e^{-\alpha \tau} \psi_{1}\left(X_{\tau}\right)\right. \\
\left.+\mathbf{1}_{\{\sigma<\tau\}} e^{-\alpha \sigma} \psi_{2}\left(X_{\sigma}\right)\right]
\end{gathered}
$$

Copyright $@$ by SIAM. Unauthorized reproduction of this article is prohibited. 
where $f, \psi_{1}, \psi_{2} \in C(E)$, and $\alpha>0$. We can define upper and lower values of the game as follows $\bar{w}(x)=\inf _{\sigma} \sup _{\tau} I_{x}(\tau, \sigma), \underline{w}(x)=\sup _{\tau} \inf _{\sigma} I_{x}(\tau, \sigma)$. We prove below that there is a value of the game and also saddle-point stopping times. We have

Theorem 3.1. For $f, \psi_{1}, \psi_{2} \in C(E)$ and $\alpha>0$ we have

$$
\bar{w}(x)=\underline{w}(x):=w(x)=I_{x}(\hat{\tau}, \hat{\sigma}),
$$

where $w \in C(E)$ and

$$
\begin{aligned}
& \hat{\tau}=\inf \left\{s \geq 0: w\left(X_{s}\right)=\psi_{1}\left(X_{s}\right)\right\} \\
& \hat{\sigma}=\inf \left\{s \geq 0: w\left(X_{s}\right)=\psi_{1}\left(X_{s}\right) \vee \psi_{2}\left(X_{s}\right)\right\}
\end{aligned}
$$

are saddle-point stopping times. Moreover

$$
m_{1}(t):=\int_{0}^{t \wedge \hat{\tau}} e^{-\alpha s} f\left(X_{s}\right) d s+e^{-\alpha(t \wedge \hat{\tau})} w\left(X_{t \wedge \hat{\tau}}\right)
$$

is a submartingale while

$$
m_{2}(t):=\int_{0}^{t \wedge \hat{\sigma}} e^{-\alpha s} f\left(X_{s}\right) d s+e^{-\alpha(t \wedge \hat{\sigma})} w\left(X_{t \wedge \hat{\sigma}}\right)
$$

is a supermartingale.

Proof. The case when $\psi_{1}(x) \leq \psi_{2}(x)$ for $x \in E$ was studied in [22] under an additional condition (A3) stated therein. Since, by the boundedness of the functions $f, \psi_{1}, \psi_{2}$, the game can be uniformly approximated by finite horizon games the proof follows now from Theorem 1 of [23]. It was shown therein that a weaker version of (A3) of [22], which is the finite-time large excursion probability of the process decays to 0 uniformly on each compact set, is sufficient and that the assumption is satisfied by Proposition 2.1 of [19]. Consequently we have the existence of the value of the game. The form of saddle-point strategies can be obtained in the same way as in [22] and [23]. The case of arbitrary $\psi_{1}, \psi_{2} \in C(E)$ was considered in Theorem 3 of [22]. We repeat simplifying the arguments of that proof here. Consider the game with cost functional

$$
\begin{aligned}
& I_{x}^{\prime}(\tau, \sigma) \\
& \quad:=E_{x}\left[\int_{0}^{\tau \wedge \sigma} e^{-\alpha s} f\left(X_{s}\right) d s+\mathbf{1}_{\{\tau \leq \sigma\}} e^{-\alpha \tau} \psi_{1}\left(X_{\tau}\right)+\mathbf{1}_{\{\sigma<\tau\}} e^{-\alpha \sigma} \psi_{1}\left(X_{\sigma}\right) \vee \psi_{2}\left(X_{\sigma}\right)\right] .
\end{aligned}
$$

This is in fact a game studied in the first part of the proof, so that there is a value $w^{\prime}(x)$ of such a game and saddle-point stopping times $\hat{\tau}^{\prime}=\inf \left\{s \geq 0: w^{\prime}\left(X_{s}\right)=\psi_{1}\left(X_{s}\right)\right\}$ and $\hat{\sigma}^{\prime}=\inf \left\{s \geq 0: w^{\prime}\left(X_{s}\right)=\psi_{1}\left(X_{s}\right) \vee \psi_{2}\left(X_{s}\right)\right\}$. Notice that for any stopping time $\sigma$

$$
I_{x}^{\prime}\left(\hat{\tau}^{\prime}, \sigma\right)=I_{x}\left(\hat{\tau}^{\prime}, \sigma\right) .
$$

In fact, we have that $\psi_{1}(x) \leq w^{\prime}(x) \leq \psi_{1}(x) \vee \psi_{2}(x)$ so that $\left\{\psi_{2}\left(X_{\sigma}\right) \leq \psi_{1}\left(X_{\sigma}\right)\right\} \subset$ $\left\{w^{\prime}\left(X_{\sigma}\right)=\psi_{1}\left(X_{\sigma}\right)\right\} \subset\left\{\hat{\tau}^{\prime} \leq \sigma\right\}$. Consequently $\left\{\sigma<\hat{\tau}^{\prime}\right\} \subset\left\{\psi_{1}\left(X_{\sigma}\right)<\psi_{2}\left(X_{\sigma}\right)\right\}$ from which (3.6) follows. For any stopping times $\tau$ and $\sigma$ using (3.6) twice and using the fact that stopping times $\hat{\tau}^{\prime}$ and $\hat{\sigma}^{\prime}$ form a saddle strategy for $I_{x}^{\prime}$ we have

$$
I_{x}\left(\tau, \hat{\sigma}^{\prime}\right) \leq I_{x}^{\prime}\left(\tau, \hat{\sigma}^{\prime}\right) \leq I_{x}^{\prime}\left(\hat{\tau}^{\prime}, \hat{\sigma}^{\prime}\right)=I_{x}\left(\hat{\tau}^{\prime}, \hat{\sigma}^{\prime}\right) \leq I_{x}^{\prime}\left(\hat{\tau}^{\prime}, \sigma\right)=I_{x}\left(\hat{\tau}^{\prime}, \sigma\right),
$$

Copyright (c) by SIAM. Unauthorized reproduction of this article is prohibited. 
which means that we have the same saddle-point stopping times for $I_{x}$ and $I_{x}^{\prime}$, and $w(x)=w^{\prime}(x)$. Sub- and super-martingale properties of (3.4) and (3.5) (resp.) follow directly from Theorem 2 of [22]. In fact, for $t, s \geq 0$, we have

$$
\begin{aligned}
& E\left[m_{1}(t+s) \mid \tilde{\mathcal{F}}_{t}\right]:=\int_{0}^{t \wedge \hat{\tau}} e^{-\alpha u} f\left(X_{u}\right) d u+\mathbf{1}_{\{\hat{\tau} \leq t\}} e^{-\alpha \hat{\tau}} w\left(X_{\hat{\tau}}\right) \\
& +\mathbf{1}_{\{t<\hat{\tau}\}} e^{-\alpha t} E\left[\int_{t}^{(t+s) \wedge \hat{\tau}} e^{-\alpha(u-t)} f\left(X_{u}\right) d u+e^{-\alpha(\hat{\tau}-t) \wedge s} w\left(X_{\hat{\tau} \wedge(t+s)}\right) \mid \tilde{\mathcal{F}}_{t}\right] \\
& \quad \geq \int_{0}^{t \wedge \hat{\tau}} e^{-\alpha u} f\left(X_{u}\right) d u+e^{-\alpha(t \wedge \hat{\tau})} w\left(X_{t \wedge \hat{\tau}}\right),
\end{aligned}
$$

where we use the fact that by Markovianity and Theorem 2 of [22] we have on the set $\{t<\hat{\tau}\}$

$$
E\left[\int_{t}^{(t+s) \wedge \hat{\tau}} e^{-\alpha(u-t)} f\left(X_{u}\right) d u+e^{-\alpha(\hat{\tau}-t) \wedge s} w\left(X_{\hat{\tau} \wedge(t+s)}\right) \mid \tilde{\mathcal{F}}_{t}\right] \geq w\left(X_{t}\right) .
$$

This implies that $m_{1}(t)$ is a submartingale. That (3.5) is a supermartingale can be proved analogously.

Remark 1. As was pointed out in Remark 2 of [23] the value of the game with the functional

$$
\begin{aligned}
& I_{x}^{\prime \prime}(\tau, \sigma) \\
& :=E_{x}\left[\int_{0}^{\tau \wedge \sigma} e^{-\alpha s} f\left(X_{s}\right) d s+\mathbf{1}_{\{\tau<\sigma\}} e^{-\alpha \tau} \psi_{1}\left(X_{\tau}\right)+\mathbf{1}_{\{\sigma \leq \tau\}} e^{-\alpha \sigma} \psi_{1}\left(X_{\sigma}\right) \vee \psi_{2}\left(X_{\sigma}\right)\right]
\end{aligned}
$$

coincides with the value $w(x)$ and is the same as in the game with the functionals $I_{x}$ and $I_{x}^{\prime}$. This is not true, however, if we consider the functional

$$
\begin{aligned}
& I_{x}^{\prime \prime \prime}(\tau, \sigma) \\
& \quad:=E_{x}\left[\int_{0}^{\tau \wedge \sigma} e^{-\alpha s} f\left(X_{s}\right) d s+\mathbf{1}_{\{\tau<\sigma\}} e^{-\alpha \tau} \psi_{1}\left(X_{\tau}\right)+\mathbf{1}_{\{\sigma \leq \tau\}} e^{-\alpha \sigma} \psi_{2}\left(X_{\sigma}\right)\right]
\end{aligned}
$$

since for $f \equiv 0, \psi_{1} \equiv 2, \psi_{2} \equiv 1$ we have $w(x)=2$, while $w^{\prime \prime \prime}(x)=\sup _{\sigma} \inf _{\tau} I_{x}^{\prime \prime \prime}(\tau, \sigma)=$ $\inf _{\tau} \sup _{\sigma} I_{x}^{\prime \prime \prime}(\tau, \sigma)=1$. One can shown (in a similar way as in the proof of Theorem $3.1)$ that the game with the functional $I_{x}^{\prime \prime \prime}$ is equivalent to the game with the functional

$$
\begin{aligned}
& I_{x}^{\prime \prime \prime \prime}(\tau, \sigma) \\
& \quad:=E_{x}\left[\int_{0}^{\tau \wedge \sigma} e^{-\alpha s} f\left(X_{s}\right) d s+\mathbf{1}_{\{\tau<\sigma\}} e^{-\alpha \tau} \psi_{1}\left(X_{\tau}\right) \wedge \psi_{2}\left(X_{\tau}\right)+\mathbf{1}_{\{\sigma \leq \tau\}} e^{-\alpha \sigma} \psi_{2}\left(X_{\sigma}\right)\right]
\end{aligned}
$$

for which we have saddle stopping times $\tilde{\tau}^{\prime}=\inf \left\{s \geq 0: w^{\prime \prime \prime}\left(X_{s}\right)=\psi_{1}\left(X_{s}\right) \wedge \psi_{2}\left(X_{s}\right)\right\}$ and $\tilde{\sigma}^{\prime}=\inf \left\{s \geq 0: w^{\prime \prime \prime}\left(X_{s}\right)=\psi_{2}\left(X_{s}\right)\right\}$.

4. Isaacs-Bellman equation and its solutions. In this section, we describe the dynamic programming formulation of our game and state the main two theorems of this paper, namely, the existence of unique continuous bounded solutions to the Isaacs-Bellman equation (Theorem 4.1) and the verification result that this solution 
is indeed the value of our game (Theorem 4.2). We also provide herein a counterexample to show that the weak assumptions made in section 2 are necessary for the uniqueness of the value function. We start with the form of Isaacs-Bellman equation corresponding to the game under (A1). Assuming that there is a value of the impulse game we have the following equation for the value function of the game:

$$
\begin{gathered}
v(x)=\inf _{\sigma} \sup _{\tau} E_{x}\left[\int_{0}^{\tau \wedge \sigma} e^{-\alpha s} f\left(X_{s}\right) d s+\mathbf{1}_{\{\tau \leq \sigma\}} e^{-\alpha \tau} M_{1}^{h} v\left(X_{\tau}\right)\right. \\
\left.+\mathbf{1}_{\{\sigma<\tau\}} e^{-\alpha \sigma} M_{2}^{h} v\left(X_{\sigma}\right)\right]
\end{gathered}
$$

with

$$
M_{1}^{h} v(x):=\sup _{\xi \in U_{1}}\left[c(x, \xi)+E_{\xi}\left[e^{-\alpha h(x, \xi)} v\left(X_{h(x, \xi)}\right)+\int_{0}^{h(x, \xi)} e^{-\alpha s} f\left(X_{s}\right) d s\right]\right]
$$

and

$$
M_{2}^{h} v(x):=\inf _{\zeta \in U_{2}}\left[d(x, \zeta)+E_{\zeta}\left[e^{-\alpha h(x, \zeta)} v\left(X_{h(x, \zeta)}\right)+\int_{0}^{h(x, \zeta)} e^{-\alpha s} f\left(X_{s}\right) d s\right]\right]
$$

where $h$ is as in (A1). By compactness of the sets $U_{1}$ and $U_{2}$ there are Borel measurable functions (selectors) $\xi^{h}: E \rightarrow U_{1}$ and $\zeta^{h}: E \rightarrow U_{2}$ such that

$M_{1}^{h} v(x):=c\left(x, \xi^{h}(x)\right)+E_{\xi^{h}(x)}\left[e^{-\alpha h\left(x, \xi^{h}(x)\right)} v\left(X_{h\left(x, \xi^{h}(x)\right)}\right)+\int_{0}^{h\left(x, \xi^{h}(x)\right)} e^{-\alpha s} f\left(X_{s}\right) d s\right]$

and

$M_{2}^{h} v(x):=d\left(x, \zeta^{h}(x)\right)+E_{\zeta^{h}(x)}\left[e^{-\alpha h\left(x, \zeta^{h}(x)\right)} v\left(X_{h\left(x, \zeta^{h}(x)\right)}\right)+\int_{0}^{h\left(x, \zeta^{h}(x)\right)} e^{-\alpha s} f\left(X_{s}\right) d s\right]$.

Under (A2) we consider a system of Isaacs-Bellman equations

$$
\begin{aligned}
v_{1}(x)=\inf _{\sigma} \sup _{\tau} E_{x}[ & \int_{0}^{\tau \wedge \sigma} e^{-\alpha s} f\left(X_{s}\right) d s+\mathbf{1}_{\{\tau \leq \sigma\}} e^{-\alpha \tau} M_{1}^{0} v_{1}\left(X_{\tau}\right) \\
& \left.+\mathbf{1}_{\{\sigma<\tau\}} e^{-\alpha \sigma} M_{2}^{0} v_{2}\left(X_{\sigma}\right)\right]
\end{aligned}
$$

and

$$
\begin{aligned}
v_{2}(x)=\inf _{\sigma} \sup _{\tau} E_{x}[ & \int_{0}^{\tau \wedge \sigma} e^{-\alpha s} f\left(X_{s}\right) d s+\mathbf{1}_{\{\tau \leq \sigma\}} e^{-\alpha \tau} M_{1}^{h} v_{1}\left(X_{\tau}\right) \\
& \left.+\mathbf{1}_{\{\sigma<\tau\}} e^{-\alpha \sigma} M_{2}^{0} v_{2}\left(X_{\sigma}\right)\right]
\end{aligned}
$$

where $M_{1}^{0}$ and $M_{2}^{0}$ are operators defined in (4.2), (4.3) (resp.) with function $h \equiv 0$. Equation (4.6) corresponds to the value of the game just after shift of the maximizer to the set $U_{1}$. The next shift by any player can be made immediately. However, by using (2.2) we shall later show that, with high probability, the next impulse time of the maximizer should be after a certain number units of time (Lemma 6.5) while the shift

Copyright (c) by SIAM. Unauthorized reproduction of this article is prohibited. 
of the minimizer can be made immediately. Equation (4.7) corresponds to the value of the game just after the shift of the minimizer to the set $U_{2}$. Again the next shift by any player can be made immediately. If this current shift by the minimizer is followed by a shift of the maximizer, then we have a decision lag of $h$ (defined in assumption (A2)) thereafter, whereas if we have another consecutive shift of the minimizer, then we have no decision lag. However, this next impulse time of the minimizer by (2.3) should, with high probability, be after a certain number units of time (Lemma 6.6).

With functions $v_{1}$ and $v_{2}$ we associate the third Isaacs-Bellman equation of the form

$$
\begin{gathered}
v(x)=\inf _{\sigma} \sup _{\tau} E_{x}\left[\int_{0}^{\tau \wedge \sigma} e^{-\alpha s} f\left(X_{s}\right) d s+\mathbf{1}_{\{\tau \leq \sigma\}} e^{-\alpha \tau} M_{1}^{0} v_{1}\left(X_{\tau}\right)\right. \\
\left.+\mathbf{1}_{\{\sigma<\tau\}} e^{-\alpha \sigma} M_{2}^{0} v_{2}\left(X_{\sigma}\right)\right] .
\end{gathered}
$$

The main results of the paper can now be formulated as follows.

THEOREM 4.1. Under (A1) or under (A2) we have a unique continuous bounded solution $v$ to (4.1) or unique continuous bounded solutions $v_{1}, v_{2}$ to the system of equations (4.6), (4.7) (resp.).

Furthermore, the following holds.

THEOREM 4.2. Under (A1) the unique solution $v$ to (4.1) or under (A2) the function $v$ defined in (4.8) determined by unique solutions to the system of equations (4.6), (4.7) is the value of the game, i.e.,

$$
v(x)=\inf _{V_{1}} \sup _{V_{2}} \mathcal{J}^{V_{1}, V_{2}}(x)=\sup _{V_{2}} \inf _{V_{1}} \mathcal{J}^{V_{1}, V_{2}}(x),
$$

and saddle-point strategies are determined as solutions to the Dynkin game formulated in (4.1) and (4.8), (4.6), (4.7), respectively.

The proof of Theorem 4.2 follows directly from Proposition 7.3, which we prove at the end of this paper, whereas the proof of Theorem 4.1 is provided under assumption (A1) in section 5 and under assumption (A2) in section 6 .

Notice first that under (A1), when $h(x, \xi) \geq h_{0}>0$ we have a contraction (in supremum norm) and therefore a unique solution to (4.1). However, we know that $h$ is a positive and continuous function and not necessarily bounded away from 0 . Therefore we have considered a finite horizon version of the game.

We now provide the following counterexample to show that the uniqueness of the value function in (4.1) may not hold without our assumptions in section 2 .

Example 1. Let $E=\{a, b\}, U_{1}=\{a\}, U_{2}=\{b\}, c(x, \xi)=-c, d(x, \zeta)=c, c>0$, $f(a)>0, f(b)<0 . X_{s}$ stays at $a(b)$ and enters $b(a)$ at a random time which is exponentially distributed with parameter $\lambda>0$. Then any function $v:\{a, b\} \mapsto R$ such that $c=v(a)-v(b)$ is a solution to (4.1) with $h \equiv 0$ which may be written as

$$
\begin{gathered}
v(x)=\inf _{\sigma} \sup _{\tau} E_{x}\left[\int_{0}^{\tau \wedge \sigma} e^{-\alpha s} f\left(X_{s}\right) d s+\mathbf{1}_{\{\tau \leq \sigma\}} e^{-\alpha \tau} M_{1}^{0} v\left(X_{\tau}\right)\right. \\
\left.+\mathbf{1}_{\{\sigma<\tau\}} e^{-\alpha \sigma} M_{1}^{0} \vee M_{2}^{0} v\left(X_{\sigma}\right)\right] .
\end{gathered}
$$

In fact, we have $M_{1}^{0} v(x)=-c+v(a), M_{2}^{0} v(x)=c+v(b)$, and clearly $M_{1}^{0} v(x)<$ $M_{2}^{0} v(x)$ for $x \in E$. When the process is in the state $a$ the minimizer shifts it immediately to the state $b$, while when the process is in the state $b$ the maximizer shifts it 
immediately to the state $a$. Consequently we have an infinite number of immediate shifts and there is an infinite number of solutions to (4.10).

Furthermore, let $v_{0}(x)=E_{x}\left[\int_{0}^{\infty} e^{-\alpha s} f\left(X_{s}\right) d s\right]$ and $v_{i}(x)$ be the value of the game with at most $i$ impulses. Consider now the case when $c<v_{0}(a)-v_{0}(b)<2 c$.

Then $v_{1}(b) \geq M_{1} v_{0}(b)>v_{0}(b)$ and $v_{1}(b)=-c+v_{0}(a)$ and the maximizer makes immediately an impulse; moreover, $v_{1}(a) \leq M_{2} v_{0}(a)=c+v_{0}(b)<v_{0}(a)$ and (since $\left.M_{1} v_{0}(x)<M_{2} v_{0}(x)\right) v_{1}(a)=c+v_{0}(b)$ so that the minimizer makes immediately an impulse.

Moreover, since $0<v_{1}(a)-v_{1}(b)=c+v_{0}(b)+c-v_{0}(a)<c$ and $v_{2}(b)=M_{1} v_{1}(v)=$ $-c+v_{1}(a)=v_{0}(b)$, and (note that we have $\left.M_{1} v_{1}(x)<M_{2} v_{1}(x)\right), v_{2}(a)=M_{2} v_{1}(a)=$ $v_{0}(a)$ and if we do not make any impulse, we obtain the same. Finally by induction we have $v_{2 i}(a)=v_{0}(a), v_{2 i}(b)=v_{0}(b) ; v_{2 i+1}(a)=c+v_{0}(b), v_{2 i+1}(b)=-c+v_{0}(a)$, and $v_{2 i+1}(a)=c+v_{2 i}(b), v_{2 i+1}(b)=-c+v_{2 i}(a)$.

5. Analysis of the impulse game under (A1). Restricting to the shifted strategies of the players (see section 2 for a description), this section proves Theorem 4.1 under the assumption (A1) of section 2. As a by-product of these proofs, we study properties of Feller-Markov processes via Lemma 5.1, which can be of independent interest. Since $\alpha>0$ and $f$ is bounded we approximate the original game by the game with the finite-horizon functional

$$
\begin{aligned}
\mathcal{J}^{V_{1}, V_{2}, T}(x) \stackrel{\text { def }}{=} E_{x}^{V_{1}, V_{2}}[ & \int_{0}^{T} e^{-\alpha s} f\left(X_{s}\right) d s \\
& \left.+\sum_{i=1}^{\infty} e^{-\alpha\left(\tau_{i} \wedge \sigma_{i}\right)}\left(\mathbf{1}_{\left\{\tau_{i} \leq \sigma_{i}\right\}} c\left(X_{\tau_{i}}^{-}, \xi_{i}\right)+\mathbf{1}_{\left\{\sigma_{i}<\tau_{i}\right\}} d\left(X_{\sigma_{i}}^{-}, \zeta_{i}\right)\right)\right] .
\end{aligned}
$$

Since $c \leq 0$ and $d \geq 0$ the players are not interested in continuing the game after time $T$. We have the following Isaacs equation,

$$
v^{T}(t, x)=0 \quad \text { for } t \geq T,
$$

and

$$
\begin{aligned}
v^{T}(t, x)=\inf _{\sigma} \sup _{\tau} E_{x}[ & \int_{0}^{\tau \wedge \sigma \wedge(T-t)} e^{-\alpha s} f\left(X_{s}\right) d s+\mathbf{1}_{\{\tau \leq \sigma\}} e^{-\alpha \tau} M_{1}^{h} v^{T}\left(t+\tau, X_{\tau}\right) \\
& \left.+\mathbf{1}_{\{\sigma<\tau\}} e^{-\alpha \sigma} M_{2}^{h} v^{T}\left(t+\sigma, X_{\sigma}\right)\right]
\end{aligned}
$$

where the operators $M_{1}^{h}, M_{2}^{h}$ are defined by (4.2), (4.3), respectively.

We shall need the following auxiliary lemmas. The first lemma is based on Proposition 2.1 of [19].

Lemma 5.1. If $P_{t} \mathcal{C}_{0} \subset \mathcal{C}_{0}$, then for any $\varepsilon>0$, compact set $U \subset E$, and $T>0$ there is $R>0$ such that

$$
\sup _{x \in U} P_{x}\left[\exists_{t \in[0, T]} \rho\left(x, X_{t}\right)>R\right] \leq \varepsilon .
$$

LEMMA 5.2. For given continuous bounded function $g$ the mapping $E \times\left(U_{1} \cup U_{2}\right) \ni$ $(x, z) \mapsto E_{z}\left[g\left(h(x, z), X_{h(x, z)}\right)\right]$ is also continuous. 
Proof. Since $h$ is a continuous function it follows directly from Lemma 2.3 of [19].

For a continuous bounded $v$ such that $v(t, x)=0$ for $t \geq T$ define the operator

$$
\begin{gathered}
S_{T} v(t, x)=\inf _{\sigma} \sup _{\tau} E_{x}\left[\int_{0}^{\tau \wedge \sigma \wedge T} e^{-\alpha s} f\left(X_{s}\right) d s+\mathbf{1}_{\{\tau \leq \sigma\}} e^{-\alpha \tau} M_{1}^{h} v\left(t+\tau, X_{\tau}\right)\right. \\
\left.+\mathbf{1}_{\{\sigma<\tau\}} e^{-\alpha \sigma} M_{2}^{h} v\left(t+\sigma, X_{\sigma}\right)\right] .
\end{gathered}
$$

Denote by $\mathcal{C}^{0}([0, T] \times E)$ the space of continuous functions $v$ such that $v(t, x)=0$ for $t \geq T$ and $x \in E$. Let

$$
h_{R}:=\inf _{x \in B\left(U_{1} \cup U_{2}, R\right), z \in U_{1} \cup U_{2}} h(x, z) .
$$

We have now the following.

Proposition 5.3. The operator $S_{T}$ is a contraction on $\mathcal{C}^{0}([0, T] \times E)$ with constant $\varepsilon+(1-\varepsilon) e^{-\alpha h_{R}}$, where $h_{R}$ is defined as in (5.5) above and $\varepsilon, R$ follows from (5.3) with $U=U_{1} \cup U_{2}$. Consequently there is a unique $v \in \mathcal{C}^{0}([0, T] \times E)$ such that $S_{T} v=v$. Moreover $v^{T}(t, x)$ is the value of impulse game restricted to shift up to time $T$ and restricted to shifted strategies defined in section 2.

Proof. Notice first that $S_{T} v(t, x)$ is the value of the stopping game starting from $t$ from $x \in E$ and with functional up to time $T$ with $\psi_{1}, \psi_{2}$ replaced by $M_{1}^{h} v$ and $M_{2}^{h} v$, respectively. From Lemma 5.2 we have continuity of the operators $M_{1}^{h}$ and $M_{2}^{h}$. Therefore using Theorem 1 of [23] (for time dependent version with $M_{1}^{h} v \leq M_{2}^{h} v$ ) and Theorem 3.1 (which shows that we can have arbitrary $M_{1}^{h} v$ and $M_{2}^{h} v$ ) we see that the operator $S_{T}$ transforms continuous bounded time space functions into itself for $t<T$. There are also no problems with time continuity of $S_{T}$ at time $T$ since, by the fact that $c$ is negative and $d$ is positive, the players after time $T$ are not interested in stopping the game before infinity. Therefore taking into account the positive discount factor $\alpha$ for $v \in \mathcal{C}^{0}([0, T] \times E)$, the function $S_{T} v$ is in $\mathcal{C}^{0}([0, T] \times E)$. For $v_{1}, v_{2} \in \mathcal{C}^{0}([0, T] \times E)$ using Lemma 5.1 with $U=U_{1} \cup U_{2}$ for a given $\varepsilon>0$ we can find $R$ such that we have (5.3) and then

$$
\begin{aligned}
& S_{T} v_{1}(t, x)-S_{T} v_{2}(t, x) \\
& \quad \leq \sup _{z \in U_{1} \cup U_{2}} \sup _{\tau} E_{x}\left\{e^{-\alpha \tau \wedge T} e^{-\alpha h\left(X_{\tau \wedge T}, z\right)}\left\|v_{1}-v_{2}\right\|\right\} \\
& \quad \leq \sup _{\tau}\left(P_{x}\left\{1_{B^{c}\left(U_{1} \cup U_{2}, R\right)}\left(X_{\tau \wedge T}\right)\right\}+E_{x}\left\{1_{B\left(U_{1} \cup U_{2}, R\right)}\left(X_{\tau \wedge T}\right) e^{-\alpha h_{R}}\right\}\right)\left\|v_{1}-v_{2}\right\| \\
& \quad \leq\left(e^{-\alpha h_{R}}+\varepsilon\left(1-e^{-\alpha h_{R}}\right)\right)\left\|v_{1}-v_{2}\right\|,
\end{aligned}
$$

where by $\|\cdot\|$ we denoted the usual supremum norm and from which the required contraction property (in this norm) follows. The proof that $v^{T}(t, x)$ is the value of the game within the class of shifted strategies follows in a standard way (see, e.g., [22] or [21], eventually [19]) using a time-dependent version of Theorem 3.1.

Remark 2. Following arguments shown later in the verification of the solution to the Isaacs-Bellman equation for the infinite horizon problem, $v^{T}(t, x)$ is also the value of the game in the case of general admissible strategies although optimal strategies are in fact in the class of shifted strategies. 
Proof of Theorem 4.1 under (A1). Notice that

$$
\left|\mathcal{J}^{V_{1}, V_{2}, T}(x)-\mathcal{J}^{V_{1}, V_{2}}(x)\right| \leq \frac{\|f\|}{\alpha} e^{-\alpha T}
$$

since both functionals differ only in the running integral term after time $T$. Consequently the solution to the finite horizon impulse game converges uniformly to the value of the infinite horizon game as $T \rightarrow \infty$. So we have that the infinite horizon game has a value and this value is a continuous function as it is a uniform limit of continuous finite horizon value functions.

6. Analysis of the impulse game under (A2). In what follows we would like to show that $v$ given by (4.8) corresponds to the value of the game when we restrict to the shifted strategies of the players (see section 2 for a description) and then prove Theorem 4.1 under the Assumption (A2) of section 2. For this purpose we study properties of Feller-Markov processes by proposing and proving Proposition 6.4, which can be of independent interest. Moreover we also propose and prove herein two important lemmas, namely, Lemmas 6.5 and 6.6, which show that consecutive jumps by any player happen with low probability. Last we also propose and prove the key Proposition 6.7, that an infinite number of jumps is not possible in finite time, which is crucial for the proof of the verification theorem 4.2 in the next section.

We consider now the following system of Isaacs-Bellman equations with decision lag $\kappa>0$ :

$$
\begin{array}{r}
v_{1}^{\kappa}(x)=\inf _{\sigma} \sup _{\tau} E_{x}\left[\int_{0}^{\tau \wedge \sigma} e^{-\alpha s} f\left(X_{s}\right) d s+\mathbf{1}_{\{\tau \leq \sigma\}} e^{-\alpha \tau} M_{1}^{\kappa} v_{1}^{\kappa}\left(X_{\tau}\right)\right. \\
\left.+\mathbf{1}_{\{\sigma<\tau\}} e^{-\alpha \sigma} M_{2}^{\kappa} v_{2}^{\kappa}\left(X_{\sigma}\right)\right]:=S_{1}^{\kappa}\left(v_{1}^{\kappa}, v_{2}^{\kappa}\right)(x)
\end{array}
$$

and

$$
\begin{aligned}
v_{2}^{\kappa}(x)=\inf _{\sigma} \sup _{\tau} E_{x}[ & \int_{0}^{\tau \wedge \sigma} e^{-\alpha s} f\left(X_{s}\right) d s+\mathbf{1}_{\{\tau \leq \sigma\}} e^{-\alpha \tau} M_{1}^{h \vee \kappa} v_{1}^{\kappa}\left(X_{\tau}\right) \\
& \left.+\mathbf{1}_{\{\sigma<\tau\}} e^{-\alpha \sigma} M_{2}^{\kappa} v_{2}^{\kappa}\left(X_{\sigma}\right)\right]:=S_{2}^{\kappa}\left(v_{1}^{\kappa}, v_{2}^{\kappa}\right)(x),
\end{aligned}
$$

defining also the operators $S^{\kappa}\left(v_{1}, v_{2}\right):=\left(S_{1}^{\kappa}\left(v_{1}, v_{2}\right), S_{2}^{\kappa}\left(v_{1}, v_{2}\right)\right)$, where, for $h^{\prime}=\kappa, h$, the operators $M_{1}^{h^{\prime}}, M_{2}^{h^{\prime}}$ correspond to $M_{1}^{h}, M_{2}^{h}$ (resp.) with $h \equiv h^{\prime}$ (see (4.2), (4.3), resp.). Notice that we have the following estimates in the supremum norm $\|\cdot\|$ :

$$
\left\|S_{1}^{\kappa}\left(v_{1}, v_{2}\right)-S_{1}^{\kappa}\left(v_{1}^{\prime}, v_{2}^{\prime}\right)\right\| \leq e^{-\alpha \kappa}\left(\left\|v_{1}-v_{1}^{\prime}\right\|+\left\|v_{2}-v_{2}^{\prime}\right\|\right)
$$

and

$$
\left\|S_{2}^{\kappa}\left(v_{1}, v_{2}\right)-S_{2}^{\kappa}\left(v_{1}^{\prime}, v_{2}^{\prime}\right)\right\| \leq e^{-\alpha \kappa}\left(\left\|v_{1}-v_{1}^{\prime}\right\|+\left\|v_{2}-v_{2}^{\prime}\right\|\right) .
$$

By Theorem 3.1, the operators $\left(S_{1}^{\kappa}, S_{2}^{\kappa}\right)$ transform $C(E) \times C(E)$ into itself since $S_{1}^{\kappa}\left(v_{1}, v_{2}\right)$ and $S_{2}^{\kappa}\left(v_{1}, v_{2}\right)$ are values of the stopping games with $\psi_{1}, \psi_{2}$ replaced by $M_{1}^{\kappa} v_{1}^{\kappa}, M_{2}^{\kappa} v_{2}^{\kappa}$ or $M_{1}^{h \vee \kappa} v_{1}^{\kappa}, M_{2}^{\kappa} v_{2}^{\kappa}$, respectively. By (6.3) and (6.4) they also form a contraction in the space $C(E) \times C(E)$. Therefore we have the following lemma.

Lemma 6.1. For each $\kappa>0$ there is a unique solution $v_{1}^{\kappa}, v_{2}^{\kappa} \in C(E) \times C(E)$ to the system of equations (6.1)-(6.2).

Copyright (C) by SIAM. Unauthorized reproduction of this article is prohibited. 
Further note that $v \in C(E)$ and $x, y \in E$ and we have

$$
\left|M_{1}^{\kappa} v(x)-M_{1}^{\kappa} v(x)\right| \leq \sup _{\xi \in U_{1}}|c(x, \xi)-c(y, \xi)|
$$

and

$$
\left|M_{2}^{\kappa} v(x)-M_{2}^{\kappa} v(x)\right| \leq \sup _{\zeta \in U_{2}}|c(x, \zeta)-c(y, \zeta)|,
$$

so that we have uniform (with respect to $\kappa$ ) continuity of $M_{1}^{\kappa} v$ and $M_{2}^{\kappa} v$. Thus we have the following theorem.

THEOREM 6.2. There is a pair of functions $\left(v_{1}, v_{2}\right) \in C(E) \times C(E)$ which are solutions to the following system of equations:

$$
\begin{aligned}
v_{1}(x)=\inf _{\sigma} \sup _{\tau} E_{x}[ & \int_{0}^{\tau \wedge \sigma} e^{-\alpha s} f\left(X_{s}\right) d s+\mathbf{1}_{\{\tau \leq \sigma\}} e^{-\alpha \tau} M_{1}^{0} v_{1}\left(X_{\tau}\right) \\
& \left.+\mathbf{1}_{\{\sigma<\tau\}} e^{-\alpha \sigma} M_{2}^{0} v_{2}\left(X_{\sigma}\right)\right]
\end{aligned}
$$

and

$$
\begin{array}{r}
v_{2}(x)=\inf _{\sigma} \sup _{\tau} E_{x}\left[\int_{0}^{\tau \wedge \sigma} e^{-\alpha s} f\left(X_{s}\right) d s+\mathbf{1}_{\{\tau \leq \sigma\}} e^{-\alpha \tau} M_{1}^{h} v_{1}\left(X_{\tau}\right)\right. \\
\left.+\mathbf{1}_{\{\sigma<\tau\}} e^{-\alpha \sigma} M_{2}^{0} v_{2}\left(X_{\sigma}\right)\right] .
\end{array}
$$

Proof. It is clear that $v_{1}^{\kappa}$ and $v_{2}^{\kappa}$ are bounded and therefore by (6.5)-(6.6) there are functions $z_{1}, z_{2} \in C(E)$ and subsequence $\kappa_{n} \rightarrow 0$ such that $M_{1}^{\kappa_{n}} v_{1}^{\kappa_{n}}(x) \rightarrow z_{1}(x)$ and $M_{2}^{\kappa_{n}} v_{2}^{\kappa_{n}}(x) \rightarrow z_{2}(x)$ uniformly in $x$ from compact subsets of $E$. Consequently

$$
\begin{aligned}
v_{1}^{\kappa_{n}}(x) \rightarrow v_{1}(x):=\inf _{\sigma} \sup _{\tau} E_{x}[ & \int_{0}^{\tau \wedge \sigma} e^{-\alpha s} f\left(X_{s}\right) d s+\mathbf{1}_{\{\tau \leq \sigma\}} e^{-\alpha \tau} z_{1}\left(X_{\tau}\right) \\
& \left.+\mathbf{1}_{\{\sigma<\tau\}} e^{-\alpha \sigma} z_{2}\left(X_{\sigma}\right)\right]
\end{aligned}
$$

uniformly on compact sets as $n \rightarrow \infty$. In fact, for a given $\varepsilon>0$ there is $T$ such that for each $n$

$$
\begin{aligned}
\mid v_{1}^{\kappa_{n}}(x)-\inf _{\sigma \leq T} \sup _{\tau \leq T} E_{x}[ & \int_{0}^{\tau \wedge \sigma} e^{-\alpha s} f\left(X_{s}\right) d s+\mathbf{1}_{\{\tau \leq \sigma\}} e^{-\alpha \tau} M_{1}^{\kappa} v_{1}^{\kappa_{n}}\left(X_{\tau}\right) \\
& \left.+\mathbf{1}_{\{\sigma<\tau\}} e^{-\alpha \sigma} M_{2}^{\kappa_{n}} v_{2}^{\kappa_{n}}\left(X_{\sigma}\right)\right] \mid \leq \varepsilon
\end{aligned}
$$

and

$$
\begin{gathered}
\mid v_{1}(x)(x)-\inf _{\sigma \leq T} \sup _{\tau \leq T} E_{x}\left[\int_{0}^{\tau \wedge \sigma} e^{-\alpha s} f\left(X_{s}\right) d s+\mathbf{1}_{\{\tau \leq \sigma\}} e^{-\alpha \tau} z_{1}\left(X_{\tau}\right)\right. \\
\left.+\mathbf{1}_{\{\sigma<\tau\}} e^{-\alpha \sigma} z_{2}\left(X_{\sigma}\right)\right] \mid \leq \varepsilon .
\end{gathered}
$$

By Lemma 5.1 and local compactness of the state space $E$ for a given compact set $K_{1}$ one can find another compact set $K_{2}$ such that for $x \in K_{1}$ we have that $\left.P_{x}\left[\exists_{t \in[0, T]} X_{t}\right) \notin K_{2}\right] \leq \varepsilon$. Since $\left\|v_{1}^{\kappa}\right\|,\left\|v_{1}^{\kappa}\right\| \leq \frac{\|f\|}{\alpha}$, functions $M_{1}^{\kappa} v_{1}^{\kappa_{n}}$ and $M_{2}^{\kappa_{n}} v_{2}^{\kappa_{n}}$ 
are uniformly bounded and the functions $z_{1}$ and $z_{2}$ are also bounded. Consequently to show the convergence (6.9) it remains to notice that uniformly in $\sigma \leq T$ and $\tau \leq T$ for $x \in K_{1}$

$$
\begin{aligned}
& E_{x}\left[\int_{0}^{\tau \wedge \sigma} e^{-\alpha s} f\left(X_{s}\right) d s+\mathbf{1}_{\{\tau \leq \sigma\}} \mathbf{1}_{X_{\tau} \in K_{2}} e^{-\alpha \tau} M_{1}^{\kappa} v_{1}^{\kappa_{n}}\left(X_{\tau}\right)\right. \\
& \left.\quad+\mathbf{1}_{\{\sigma<\tau\}} \mathbf{1}_{X_{\sigma} \in K_{2}} e^{-\alpha \sigma} M_{2}^{\kappa_{n}} v_{2}^{\kappa_{n}}\left(X_{\sigma}\right)\right] \rightarrow \\
& E_{x}\left[\int_{0}^{\tau \wedge \sigma} e^{-\alpha s} f\left(X_{s}\right) d s+\mathbf{1}_{\{\tau \leq \sigma\}} \mathbf{1}_{X_{\tau} \in K_{2}} e^{-\alpha \tau} z_{1}\left(X_{\tau}\right)\right. \\
& \left.\quad+\mathbf{1}_{\{\sigma<\tau\}} \mathbf{1}_{X_{\sigma} \in K_{2}} e^{-\alpha \sigma} z_{2}\left(X_{\sigma}\right)\right]
\end{aligned}
$$

which completes the proof of (6.9).

We also have

$$
M_{1}^{h \vee \kappa_{n}} v_{1}^{\kappa_{n}}(x) \rightarrow M_{1}^{h} v_{1}(x)
$$

uniformly in $x$ from compact sets as $n \rightarrow \infty$. Repeating the arguments as in (6.9) we obtain that

$$
\begin{aligned}
v_{2}^{\kappa_{n}}(x) \rightarrow v_{2}(x):=\inf _{\sigma} \sup _{\tau} E_{x}[ & \int_{0}^{\tau \wedge \sigma} e^{-\alpha s} f\left(X_{s}\right) d s+\mathbf{1}_{\{\tau \leq \sigma\}} e^{-\alpha \tau} M_{1}^{h} v_{1}\left(X_{\tau}\right) \\
& \left.+\mathbf{1}_{\{\sigma<\tau\}} e^{-\alpha \sigma} z_{2}\left(X_{\sigma}\right)\right]
\end{aligned}
$$

also uniformly on compact sets. Therefore we have that $M_{1}^{\kappa_{n}} v_{1}^{\kappa_{n}}(x) \rightarrow M_{1}^{0} v_{1}(x)$ and $M_{2}^{\kappa_{n}} v_{2}^{\kappa_{n}}(x) \rightarrow M_{2}^{0} v_{2}(x)$ uniformly in $x$ from compact sets as $n \rightarrow \infty$, which means that $v_{1}$ and $v_{2}$ are solutions to the system of equations (6.7)-(6.8).

We now recall the following result from Theorem 3.7 of [6].

Lemma 6.3. For any compact set $K \subseteq E$ and any $\varepsilon, \delta>0$ there is $\kappa_{0}>0$ such that

$$
\sup _{0 \leq \kappa \leq \kappa_{0}} \sup _{x \in K} P_{x}\{X(\kappa) \notin B(x, \delta)\}<\varepsilon .
$$

Using Lemma 5.1 we obtain a stronger version of the last lemma.

Proposition 6.4. For any compact set $K \subset E$ and $\delta>0$

$$
\lim _{\kappa \rightarrow 0} \sup _{x \in K} P_{x}\left\{\exists_{s \in[0, \kappa]}: \rho\left(x, X_{s}\right) \geq \delta\right\} \rightarrow 0 .
$$

Proof. Let $x \in K$ a compact set and $\delta>0$. By Lemma 5.1 for a given $\varepsilon>0$ there is $R>3 \delta$ such that $\sup _{x \in K} P_{x}\left[\exists_{t \in[0, T]} \rho\left(x, X_{t}\right)>R\right] \leq \varepsilon$. By Lemma 6.3 there is $\kappa_{0}$ such that for $\kappa \leq \kappa_{0}$ we have $\sup _{x \in B(K, R)} P_{x}\{X(\kappa) \notin B(x, \delta)\}<\varepsilon$. Let $\tau:=\inf \left\{s \geq 0: \rho\left(x, X_{s}\right)>3 \delta\right\}$. Then we have

$$
\begin{aligned}
1-\varepsilon & \leq P_{x}\left\{\rho\left(x, X_{\kappa}\right) \leq \delta\right\} \leq P_{x}\left\{\rho\left(x, X_{\kappa}\right) \leq \delta, \tau \leq \kappa\right\}+P_{x}\{\tau>\kappa\} \\
& \leq \varepsilon+E_{x}\left\{1_{\tau \leq \kappa} 1_{\rho\left(x, X_{\tau}\right) \leq R} P_{X_{\tau}}\left\{\rho\left(x, X_{\kappa-\tau}\right) \leq \delta\right\}\right\}+P_{x}\{\tau>\kappa\} \\
& \leq \varepsilon+\varepsilon P_{x}\{\tau \leq \kappa\}+P_{x}\{\tau>\kappa\}=\varepsilon+\varepsilon+P_{x}\{\tau>\kappa\}(1-\varepsilon)
\end{aligned}
$$

Copyright $@$ by SIAM. Unauthorized reproduction of this article is prohibited. 
since $\left\{\rho\left(x, X_{\kappa}\right) \leq \delta\right\} \subset\left\{\rho\left(X_{\tau}, X_{\kappa}\right) \geq 2 \delta\right\} \subset\left\{\rho\left(X_{\tau}, X_{\kappa}\right)>\delta\right\}$, so that on $\{\tau \leq \kappa\}$

$$
1_{\rho\left(x, X_{\tau}\right) \leq R} P_{X_{\tau}}\left\{\rho\left(x, X_{\kappa-\tau}\right) \leq \delta\right\} \leq \varepsilon
$$

and consequently $P_{x}\{\tau>\kappa\} \geq \frac{1-3 \varepsilon}{1-\varepsilon}$, which taking into account that $\varepsilon$ could be chosen arbitrarily small completes the proof.

Fix $r>0$. By (2.2) and (2.3), respectively, there is $\varepsilon>0$ such that, respectively,

$$
\forall_{x \in B\left(U_{1}, r\right)} \forall_{\xi, \xi^{\prime} \in U_{1}} c(x, \xi)+c\left(\xi, \xi^{\prime}\right)<c\left(x, \xi^{\prime}\right)-3 \varepsilon
$$

and

$$
\forall_{x \in B\left(U_{2}, r\right)} \forall_{\zeta, \zeta^{\prime} \in U_{2}} d\left(x, \zeta^{\prime}\right)+3 \varepsilon<d(x, \zeta)+d\left(\zeta, \zeta^{\prime}\right) .
$$

There is $\delta>0$ such that $\delta \leq r$ and for $x \in B\left(U_{1}, r\right)$

$$
\forall_{\xi, \xi^{\prime} \in U_{1}} \forall_{\xi^{\prime \prime} \in B(\xi, \delta)} c(x, \xi)+c\left(\xi^{\prime \prime}, \xi^{\prime}\right)<c\left(x, \xi^{\prime}\right)-2 \varepsilon
$$

while for $x \in B\left(U_{2}, r\right)$

$$
\forall_{\zeta, \zeta^{\prime} \in U_{2}} \forall_{\zeta^{\prime \prime} \in B(\zeta, \delta)} d\left(x, \zeta^{\prime}\right)+2 \varepsilon<d(x, \zeta)+d\left(\zeta^{\prime \prime}, \zeta^{\prime}\right) .
$$

For if, say, (6.19) is not true, then there are sequences $x_{n} \in B\left(U_{1}, r\right), \xi_{n}^{(1)}, \xi_{n}^{(2)} \in$ $U_{1}, \xi_{n}^{\prime \prime} \in B\left(\xi_{n}^{(2)}, \frac{1}{n}\right)$ such that $c\left(x_{n}, \xi_{n}^{(2)}+c\left(\xi_{n}^{\prime \prime}, \xi_{n}^{(1)}\right)+\frac{1}{n} \geq c\left(x_{n}, \xi_{n}^{(1)}\right)\right.$ and hence choosing suitable convergent subsequences we have that $x_{n_{k}} \longrightarrow x, \xi_{n_{k}}^{(1)} \longrightarrow \xi^{\prime}, \xi_{n_{k}}^{(2)} \longrightarrow$ $\xi, \xi_{n_{k}}^{\prime \prime} \longrightarrow \xi$, thus obtaining $c(x, \xi)+c\left(\xi, \xi^{\prime}\right) \geq c\left(x, \xi^{\prime}\right)$, which is a contradiction to (2.2). Similarly (6.20) holds, otherwise contradicting (2.3).

By the continuity of $v_{1}(\cdot)$ and $v_{2}(\cdot)$ (see Theorem 6.2) we have that there is $\delta^{\prime}>0$ such that $\delta^{\prime} \leq \delta$ and

$$
\sup _{\xi \in U_{1}} \sup _{z \in B\left(\xi, \delta^{\prime}\right)}\left|v_{1}(\xi)-v_{1}(z)\right| \leq \varepsilon
$$

and

$$
\sup _{\zeta \in U_{2}} \sup _{z \in B\left(\zeta, \delta^{\prime}\right)}\left|v_{2}(\zeta)-v_{2}(z)\right| \leq \varepsilon
$$

Let $\bar{h}=\inf _{x \in B\left(U_{2}, r\right), \xi \in U_{1}} h(x, \xi)$. By Proposition 6.4 for each $\tilde{\varepsilon}>0$ there is $\tilde{h}>0$ such that $\tilde{h} \leq \bar{h}$ and

$$
\sup _{x \in U_{1} \cup U_{2}} P_{x}\left\{\exists_{s \in[0, \tilde{h}]} \rho\left(X_{s}, x\right) \geq \delta^{\prime}\right\}<\tilde{\varepsilon} .
$$

In what follows we shall assume, without loss of generality, that $\tilde{\varepsilon}<\frac{1}{2}$. Denote by $\xi: E \rightarrow U_{1}$ and $\zeta: E \rightarrow U_{2}$ Borel measurable functions (selectors) such that for $z \in E$

$$
M_{1}^{0} v_{1}(z)=c(z, \xi(z))+v_{1}(\xi(z)) \quad \text { and } \quad \mathcal{M}_{2}^{0} v_{2}(z)=d(z, \zeta(z))+v_{2}(\zeta(z)) .
$$

Let

$(6.25)$

$\Gamma_{1}=\left\{z \in E: v_{1}(z)=M_{1}^{0} v_{1}(z)\right\} \quad$ and $\quad \Gamma_{2}=\left\{z \in E: v_{2}(z)=M_{2}^{0} v_{2}(z) \vee M_{1}^{h} v_{1}(z)\right\}$.

The next two important lemmas show that two successive impulses by the minimizer or the maximizer can happen only with a small probability.

Copyright ( $)$ by SIAM. Unauthorized reproduction of this article is prohibited. 
Lemma 6.5. Assume that $z \in \Gamma_{1} \cap B\left(U_{1}, r\right)$ and $\tau^{*}=\inf \left\{s \geq 0: \bar{X}_{s}^{1} \in \Gamma_{1}\right\}$, where $\left(\bar{X}_{s}^{1}\right)$ is a copy of Markov process $\left(X_{s}\right)$ starting from $\bar{X}_{0}^{1}=\xi(z)$. Then for $\tilde{h}$ as in (6.23) we have

$$
P_{\xi(z)}\left\{\tau^{*} \leq \tilde{h}\right\}<\tilde{\varepsilon}
$$

Proof. Assuming $\rho\left(\bar{X}_{0}^{1}, \bar{X}_{\tau^{*}}^{1}\right)<\delta^{\prime}$ we have using (6.21) and (6.19)

$$
\begin{aligned}
v_{1}(z) & =M_{1}^{0} v_{1}(z)=c(z, \xi(z))+v_{1}(\xi(z)) \leq c(z, \xi(z))+v_{1}\left(\bar{X}_{\tau^{*}}^{1}\right)+\varepsilon \\
& =c(z, \xi(z))+c\left(\bar{X}_{\tau^{*}}^{1}, \xi\left(\bar{X}_{\tau^{*}}^{1}\right)\right)+v_{1}\left(\xi\left(\bar{X}_{\tau^{*}}^{1}\right)\right)+\varepsilon \\
& \leq c\left(z, \xi\left(\bar{X}_{\tau^{*}}^{1}\right)\right)-2 \varepsilon+v_{1}\left(\xi\left(\bar{X}_{\tau^{*}}^{1}\right)\right)+\varepsilon \leq M_{1}^{0} v_{1}(z)-\varepsilon,
\end{aligned}
$$

which leads to a contradiction since $v_{1}(z)=M_{1}^{0} v_{1}(z)$ as $z \in \Gamma_{1}$. Therefore we should have $\rho\left(\bar{X}_{0}^{1}, \bar{X}_{\tau^{*}}^{1}\right) \geq \delta^{\prime}$ and by (6.23) we have that (6.26) holds, which completes the proof.

Lemma 6.6. Assume that $z \in \Gamma_{2} \cap B\left(U_{2}, r\right)$ and $\sigma^{*}=\inf \left\{s \geq 0: \bar{X}_{s}^{1} \in \Gamma_{2}\right\}$, where $\left(\bar{X}_{s}^{1}\right)$ is a copy of Markov process $\left(X_{s}\right)$ starting from $\bar{X}_{0}^{1}=\zeta(z)$. Then either at time 0 or at time $\sigma^{*}$ we have impulse with decision lag at least $\bar{h}$, or for $\tilde{h}$ as in (6.23) we have

$$
P_{\zeta(z)}\left\{\sigma^{*} \leq \tilde{h}\right\}<\tilde{\varepsilon}
$$

Proof. When $\rho\left(\bar{X}_{0}^{1}, \bar{X}_{\sigma^{*}}^{1}\right)<\delta^{\prime}$ and $v_{2}(z)=M_{2}^{0} v_{2}(z)$ we have using (6.22)

$$
v_{2}(z)=M_{2}^{0} v_{2}(z)=d(z, \zeta(z))+v_{2}(\zeta(z)) \geq d(z, \zeta(z))+v_{2}\left(\bar{X}_{\sigma^{*}}^{1}\right)-\varepsilon .
$$

When additionally $v_{2}\left(\bar{X}_{\sigma^{*}}^{1}\right)=M_{2}^{0} v_{2}\left(\bar{X}_{\sigma^{*}}^{1}\right)$ then continuing (6.28) and using (6.20) we obtain

$$
\begin{aligned}
& v_{2}(z) \geq d(z, \zeta(z))+d\left(\bar{X}_{\sigma^{*}}^{1}, \zeta\left(\bar{X}_{\sigma^{*}}^{1}\right)\right)+v_{2}\left(\zeta\left(\bar{X}_{\sigma^{*}}^{1}\right)\right)-\varepsilon \\
& >d\left(z, \zeta\left(\bar{X}_{\sigma^{*}}^{1}\right)\right)+v_{2}\left(\zeta\left(\bar{X}_{\sigma^{*}}^{1}\right)\right)+2 \varepsilon-\varepsilon \geq M_{2}^{0} v_{2}(z)+\varepsilon,
\end{aligned}
$$

which leads to contradiction since $v^{2}(z)=M_{2}^{0} v_{2}(z)$ as $z \in \Gamma_{2}$. Therefore either $v_{2}(z)=M_{1}^{h} v_{1}(z)$ or $v_{2}\left(\bar{X}_{\sigma^{*}}^{1}\right)=M_{1}^{h} v_{1}\left(\bar{X}_{\sigma^{*}}^{1}\right)$ or $\rho\left(\bar{X}_{0}^{1}, \bar{X}_{\sigma^{*}}^{1}\right) \geq \delta^{\prime}$. In the first two cases we have impulses with decision lag $h \geq \bar{h}$ and in the third case by (6.23) we have (6.27).

Let $\xi^{h}$ be (by analogy to (4.4)) a Borel measurable function (selector) $\xi^{h}: E \rightarrow U_{1}$ such that

$M_{1}^{h} v_{1}(x):=c\left(x, \xi^{h}(x)\right)+E_{\xi^{h}(x)}\left[e^{-\alpha h\left(x, \xi^{h}(x)\right)} v_{1}\left(X_{h\left(x, \xi^{h}(x)\right)}\right)+\int_{0}^{h\left(x, \xi^{h}(x)\right)} e^{-\alpha s} f\left(X_{s}\right) d s\right]$.

We recall here that $\left(\bar{X}_{s}^{i}\right)$ below are copies of the Markov process $\left(X_{s}\right)$ starting at $\rho_{i-1}$ from the state defined by our impulsive strategy, while $\left(X_{s}^{i}\right)$ is our controlled process between $(i-1)$ th and $i$ th impulse, introduced at the beginning of the paper when we constructed our controlled probability space. Define now the sequence $\rho_{i}^{*}$ of stopping times inductively by the following formulae:

$$
\begin{aligned}
& \rho_{1}^{*}:=\tau^{1 *} \wedge \sigma^{1 *}, \text { where } \\
& \tau^{1 *}:=\inf \left\{s \geq 0: v\left(\bar{X}_{s}^{1}\right)=M_{1}^{0} v_{1}\left(\bar{X}_{s}^{1}\right)\right\}
\end{aligned}
$$


$\sigma^{1 *}:=\inf \left\{s \geq 0: v\left(\bar{X}_{s}^{1}\right)=M_{2}^{0} v_{2}\left(\bar{X}_{s}^{1}\right) \vee M_{1}^{0} v_{1}\left(\bar{X}_{s}^{1}\right)\right\}$,

$\rho_{2}^{*}:=\rho_{1}^{*}+\left(\tau^{2 *} \wedge \sigma^{2 *}\right) \circ \theta_{\rho_{1}^{*}}$

and when $\rho_{1}^{*}=\tau^{1 *} \leq \sigma^{*}$ we have

$\bar{X}_{0}^{2}=\xi\left(\bar{X}_{\rho_{1}^{*}}^{1}\right)$,

$\tau^{2 *}:=\inf \left\{s \geq 0: v_{1}\left(\bar{X}_{s}^{2}\right)=M_{1}^{0} v_{1}\left(\bar{X}_{s}^{2}\right)\right\}$,

$\sigma^{2 *}:=\inf \left\{s \geq 0: v_{1}\left(\bar{X}_{s}^{2}\right)=M_{2}^{0} v_{2}\left(\bar{X}_{s}^{2}\right) \vee M_{1}^{0} v_{1}\left(\bar{X}_{s}^{2}\right)\right\}$,

while when $\rho_{1}^{*}=\sigma^{1 *}<\tau^{1 *}$ we have

$\bar{X}_{0}^{2}=\zeta\left(X_{\rho_{1}^{*}}^{1}\right)$,

where, this time, $\tau^{2 *}:=\inf \left\{s \geq 0: v_{2}\left(\bar{X}_{s}^{2}\right)=M_{1}^{h} v_{1}\left(\bar{X}_{s}^{2}\right)\right\}$

and $\sigma^{2 *}:=\inf \left\{s \geq 0: v_{2}\left(\bar{X}_{s}^{2}\right)=M_{2}^{0} v_{2}\left(\bar{X}_{s}^{2}\right) \vee M_{1}^{h} v_{1}\left(\bar{X}_{s}^{2}\right)\right\}$

and finally when $\rho_{2}^{*}=\rho_{1}^{*}+\tau^{2 *} \circ \theta_{\rho_{1}^{*}}$ the decision lag of $h\left(X_{\rho_{2}^{*}}^{2}, \xi^{h}\left(X_{\rho_{2}^{*}}^{2}\right)\right)$ is executed

so that the next shift is allowed after $\rho_{2}^{*}+h\left(X_{\rho_{2}^{*}}^{2}, \xi^{h}\left(X_{\rho_{2}^{*}}^{2}\right)\right)$.

In the $i$ th iteration we have the following five cases:

1. Given $\rho_{i}^{*}=\rho_{i-1}^{*}+\tau^{i *} \circ \theta_{\rho_{i-1}^{*}}$, where $\tau^{i *}=\inf \left\{s \geq 0: v_{1}\left(\bar{X}_{s}^{i}\right)=M_{1}^{0} v_{1}\left(\bar{X}_{s}^{i}\right)\right\}$, which means that we had an impulse of the player I (maximizer) and we are just after another impulse of the player I and follow (6.7). Define $\rho_{i+1}^{*}:=$ $\rho_{i}^{*}+\left(\tau^{(i+1) *} \wedge \sigma^{(i+1) *}\right) \circ \theta_{\rho_{i}^{*}}$, where $\bar{X}^{i+1}$ starts from $\xi\left(X_{\tau^{i *}}^{i}\right)$ and $\tau^{(i+1) *}:=$ $\inf \left\{s \geq 0: v_{1}\left(\bar{X}_{s}^{i+1}\right)=M_{1}^{0} v_{1}\left(\bar{X}_{s}^{i+1}\right)\right\}$ and $\sigma^{(i+1) *}:=\inf \left\{s \geq 0: v_{1}\left(\bar{X}_{s}^{i+1}\right)=\right.$ $\left.M_{2}^{0} v_{2}\left(\bar{X}_{s}^{i+1}\right) \vee M_{1}^{0} v_{1}\left(\bar{X}_{s}^{i+1}\right)\right\}$.

2. Given $\rho_{i}^{*}=\rho_{i-1}^{*}+\sigma^{i *} \circ \theta_{\rho_{i-1}^{*}}$, where $\sigma^{i *}:=\inf \left\{s \geq 0: v_{1}\left(\bar{X}_{s}^{i}\right)=M_{2}^{0} v_{2}\left(\bar{X}_{s}^{i}\right) \vee\right.$ $\left.M_{1}^{0} v_{1}\left(\bar{X}_{s}^{i}\right)\right\}$ and $v_{1}\left(X_{\rho_{i}^{*}}^{i}\right)<M_{1}^{0} v_{1}\left(X_{\rho_{i}^{*}}^{i}\right)$, which means that we had an impulse of the player I and we are now after the impulse of the player II (minimizer) and follow (6.8). Define $\rho_{i+1}^{*}:=\rho_{i}^{*}+\left(\tau^{(i+1) *} \wedge \sigma^{(i+1)^{*}}\right) \circ \theta_{\rho_{i}^{*}}$ with $\tau^{(i+1)^{*}}:=$ $\inf \left\{s \geq 0: v_{2}\left(\bar{X}_{s}^{i+1}\right)=M_{1}^{h} v_{1}\left(\bar{X}_{s}^{i+1}\right)\right\}, \sigma^{(i+1)^{*}}:=\inf \left\{s \geq 0: v_{2}\left(\bar{X}_{s}^{i+1}\right)=\right.$ $\left.M_{2}^{0} v_{2}\left(\bar{X}_{s}^{i+1}\right) \vee M_{1}^{h} v_{1}\left(\bar{X}_{s}^{i+1}\right)\right\}$, where $\bar{X}^{i+1}$ starts from $\bar{\zeta}\left(X_{\tau^{i *}}^{i}\right)$ and when $\rho_{i+1}^{*}:=\rho_{i}^{*}+\tau^{(i+1) *} \circ \theta_{\rho_{i}^{*}}$ we have decision lag $h\left(X_{\rho_{i+1}^{*}}^{i+1}, \xi^{h}\left(X_{\rho_{i+1}^{*}}^{i+1}\right)\right)$ at time $\rho_{i+1}^{*}$, i.e., the next impulse is after $\rho_{i+1}^{*}+h\left(X_{\rho_{i+1}^{*}}^{i+1}, \xi^{h}\left(X_{\rho_{i+1}^{*}}^{i+1}\right)\right)$.

3. Given $\rho_{i}^{*}=\rho_{i-1}^{*}+h\left(X_{\rho_{i-1}}^{i}, \xi^{h}\left(X_{\rho_{i-1}}^{i}\right)\right)+\tau^{i *} \circ \theta_{\rho_{i-1}^{*}+h\left(X_{\rho_{i-1}}^{i}, \xi^{h}\left(X_{\rho_{i-1}}^{i}\right)\right)}$, where $\tau^{i *}=\inf \left\{s \geq 0: v_{2}\left(\bar{X}_{s}^{i}\right)=M_{1}^{h} v_{1}\left(\bar{X}_{s}^{i}\right)\right\}$, which means that after the impulse of the minimizer we have an impulse of the maximizer and therefore we have decision lag and then another impulse of the maximizer and follow (6.7). Define $\rho_{i+1}^{*}:=\rho_{i}^{*}+\left(\tau^{(i+1) *} \wedge \sigma^{(i+1) *}\right) \circ \theta_{\rho_{i}^{*}}$, where $\tau^{(i+1) *}:=\inf \{s \geq$ $\left.0: v_{1}\left(\bar{X}_{s}^{i+1}\right)=M_{1}^{0} v_{1}\left(\bar{X}_{s}^{i+1}\right)\right\}$ and $\sigma^{(i+1) *}:=\inf \left\{s \geq 0: v_{1}\left(\bar{X}_{s}^{i+1}\right)=\right.$ $\left.M_{2}^{0} v_{2}\left(\bar{X}_{s}^{i+1}\right) \vee M_{1}^{0} v_{1}\left(\bar{X}_{s}^{i+1}\right)\right\}$ and where $\bar{X}^{i+1}$ starts from $\xi\left(X_{\tau^{i *}}^{i}\right)$.

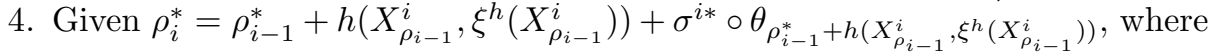
$\sigma^{i *}=\inf _{2}\left\{s \geq 0: v_{1}\left(\bar{X}_{s}^{i}\right)=M_{2} v_{2}\left(\bar{X}_{s}^{i}\right) \vee M_{1}^{h} v_{1}\left(\bar{X}_{s}^{i}\right)\right\}$, when $v_{1}\left(\bar{X}_{\sigma^{i *}}^{i}\right)<$ $M_{1}^{h} v_{1}\left(\bar{X}_{\sigma^{i *}}^{i}\right)$, which means that after an impulse of the minimizer we have an impulse of the maximizer and therefore we have decision lag and then another impulse of the minimizer and follow (6.8). Define $\rho_{i+1}^{*}:=\rho_{i}^{*}+$ $\left(\tau^{(i+1) *} \wedge \sigma^{(i+1) *}\right) \circ \theta_{\rho_{i}^{*}}$, with $\tau^{(i+1) *}:=\inf \left\{s \geq 0: v_{2}\left(\bar{X}_{s}^{i+1}\right)=M_{1}^{h} v_{1}\left(\bar{X}_{s}^{i+1}\right)\right\}$ and $\sigma^{(i+1) *}:=\inf \left\{s \geq 0: v_{2}\left(\bar{X}_{s}^{i+1}\right)=M_{2}^{0} v_{2}\left(\bar{X}_{s}^{i+1}\right) \vee M_{1}^{h} v_{1}\left(\bar{X}_{s}^{i+1}\right)\right\}$, where $\bar{X}^{i+1}$ starts from the state of Markov process $\zeta\left(X_{\sigma^{i *}}^{i}\right)$ and when $\rho_{i+1}^{*}:=$ $\rho_{i}^{*}+\tau^{(i+1) *} \circ \theta_{\rho_{i}^{*}}$ we have decision lag $h\left(X_{\rho_{i+1}^{*}}^{i+1}, \xi^{h}\left(X_{\rho_{i+1}^{*}}^{i+1}\right)\right)$ at time $\rho_{i+1}^{*}$.

5. Given $\rho_{i}^{*}=\rho_{i-1}^{*}+\sigma^{i *} \circ \theta_{\rho_{i-1}^{*}}$, where $\sigma^{i *}:=\inf \left\{s \geq 0: v_{2}\left(\bar{X}_{s}^{i}\right)=M_{2}^{0} v_{2}\left(\bar{X}_{s}^{i}\right) \vee\right.$ $\left.M_{1}^{h} v_{1}\left(\bar{X}_{s}^{i}\right)\right\}$ and $v_{2}\left(\bar{X}_{\rho_{i}^{*}}^{i}\right)<M_{1}^{h} v_{1}\left(\bar{X}_{\rho_{i}^{*}}^{i}\right)$, which means that after an impulse of the minimizer we have another impulse of the minimizer and follow (6.8). De- 


$$
\begin{aligned}
& \text { fine } \rho_{i+1}^{*}:=\rho_{i}^{*}+\left(\tau^{(i+1) *} \wedge \sigma^{(i+1) *}\right) \circ \theta_{\rho_{i}^{*}}, \text { with } \tau^{(i+1) *}:=\inf \left\{s \geq 0: v_{2}\left(\bar{X}_{s}^{i+1}\right)=\right. \\
& \left.M_{1}^{h} v_{1}\left(\bar{X}_{s}^{i+1}\right)\right\}, \sigma^{(i+1) *}:=\inf \left\{s \geq 0: v_{2}\left(\bar{X}_{s}^{i+1}\right)=M_{2}^{0} v_{2}\left(\bar{X}_{s}^{i+1}\right) \vee M_{1}^{h} v_{1}\left(\bar{X}_{s}^{i+1}\right)\right\}, \\
& \text { where } \bar{X}^{i+1} \text { starts from } \zeta\left(X_{\sigma^{i *}}^{i}\right) .
\end{aligned}
$$

We can now define strategies $V_{1}^{*}:=\left(\hat{\tau}_{1}, \hat{\xi}_{1} ; \hat{\tau}_{2}, \hat{\xi}_{2} ; \ldots\right), V_{2}^{*}:=\left(\hat{\sigma}_{1}, \hat{\zeta}_{1} ; \hat{\sigma}_{2}, \hat{\zeta}_{2} ; \ldots\right)$ of the players I and II, respectively, by $\hat{\tau}_{1}=\tau^{1 *}, \hat{\xi}_{1}=\xi\left(X_{\hat{\tau}_{1}}^{1}\right), \hat{\sigma}_{1}=\sigma^{1 *}, \hat{\zeta}_{1}=\zeta\left(X_{\hat{\sigma}_{1}}^{1}\right)$, and for $i \geq 0, \hat{\tau}_{i+1}=\rho_{i}^{*}+\tau^{(i+1) *} \circ \theta_{\rho_{i}^{*}}, \hat{\xi}_{i+1}=\xi\left(X_{\hat{\tau}_{i+1}}^{i+1}\right)$ or $\hat{\xi}_{i+1}=\xi^{h}\left(X_{\hat{\tau}_{i+1}}^{i+1}\right)$ whenever at time $\hat{\tau}_{i}$ we had an impulse of the minimizer, and $\hat{\sigma}_{i+1}=\rho_{i}^{*}+\sigma^{(i+1) *} \circ \theta_{\rho_{i}^{*}}, \hat{\zeta}_{i+1}=$ $\zeta\left(X_{\hat{\sigma}_{i+1}}^{i+1}\right)$. The following key result will be crucial for verification of the Bellman system (4.6)-(4.8).

Proposition 6.7. We have that $\rho_{n}^{*} \rightarrow \infty$ as $n \rightarrow \infty, P_{x}^{V_{1}^{*}, V_{2}^{*}}$ almost surely.

Proof. Assume that $\rho_{n}^{*} \rightarrow \rho^{*} \in[0, \infty]$, as $n \rightarrow \infty$ and for $A:=\left\{\rho^{*}<\infty\right\}$ we have that $P_{x}^{V_{1}^{*}, V_{2}^{*}}(A)=\gamma>0$. Let $A_{n}=\left\{\rho^{*}-\rho_{n}^{*}<\tilde{h}\right\} \cap A$. Clearly $A_{n} \subset A$, $A_{n} \subset A_{n+1}$, and $\cup_{n=1}^{\infty} A_{n}=A$. Therefore there is $N$ such that for $n \geq N$ we have that $P_{x}^{V_{1}^{*}, V_{2}^{*}}\left(A_{n}\right) \geq \frac{\gamma}{2}$.

By (6.23) after each impulse the probability that the controlled process leaves $B\left(U_{1} \cup U_{2}, r\right)$ within $\tilde{h}$ units of time is less than $\tilde{\varepsilon}$. If it does not leave $B\left(U_{1} \cup U_{2}, r\right)$ within $\tilde{h}$ units of time, then every repeated impulse by the same player can happen (within $\tilde{h}$ units of time) with probability at most $\tilde{\varepsilon}$. Consequently with probability at most $\tilde{\varepsilon}+\tilde{\varepsilon}=2 \tilde{\varepsilon}<1$ within $\tilde{h}$ units of time we have no two repeated impulses by the same player. Therefore for each $n \geq N$ after the $n$th impulse we have no two repeated impulses (within $\tilde{h}$ units of time) with probability at most $2 \tilde{\varepsilon}<1$. If we have $k+1$, $k=1,2, \ldots$ successive repeated impulses by the same player (all within $\tilde{h}$ units of time), then it happens with probability at most $(2 \tilde{\varepsilon})^{k}$. Therefore after $n$ impulses we have either another impulse after $\tilde{h}$ units of time, or we have successive shifts of the same player. When there is a change of the player who exercises impulses then in the case of an impulse of the change from maximizer to minimizer we have no transaction lag, while in the case of the change from minimizer to maximizer we have decision lag which is greater than $\tilde{h}$ with probability at least $1-\tilde{\varepsilon}$ (with such probability the controlled process does not leave $B\left(U_{2}, r\right)$ within $\tilde{h}$ units of time). Summing up we cannot have $\gamma>0$, since otherwise the probability that we have impulses within $\tilde{h}$ units of time decreases to 0 , which gives us a contradiction.

Proof of Theorem 4.1 under (A2). By Theorem 6.2 we are given a solution $v, v_{1}, v_{2}$ to the system of Bellman equations (4.8), (4.6), (4.7). By Theorem 3.1 each solution equation corresponds to the value function in the stopping game with suitable functions $\psi_{1}$ and $\psi_{2}$, for which we have exact formulae for saddle-point stopping times. In the case when we are using shifted strategies practically we solve at each random time $\rho_{i}^{*}$ a new game with strategies not depending on the past (before $\rho_{i}$ ). In particular, when $\rho_{i}^{*}=\rho_{i-1}^{*}+\tau_{i}^{*} \circ \theta_{\rho_{i-1}^{*}}$, we have, by Theorem 3.1,

$$
\begin{aligned}
v_{1}\left(X_{\rho_{i}^{*}}^{i}\right)=E_{X_{\rho_{i}^{*}}^{i}}\left[\int_{0}^{\tau^{(i+1) *} \wedge \sigma^{(i+1) *}} e^{-\alpha s} f\left(X_{s}^{i+1}\right) d s\right. & \\
& +\mathbf{1}_{\left\{\tau^{(i+1) *} \leq \sigma^{(i+1) *}\right\}} e^{-\alpha \tau^{(i+1) *}} M_{1}^{0} v_{1}\left(X_{\left.\tau^{(i+1) *}\right)}\right. \\
& \left.+\mathbf{1}_{\left\{\sigma^{(i+1) *}<\tau^{(i+1) *}\right\}} e^{-\alpha \sigma^{(i+1) *}} M_{2}^{0} v_{2}\left(X_{\sigma^{(i+1) *}}\right)\right],
\end{aligned}
$$


while whenever $\rho_{i}^{*}=\sigma^{i *} \circ \theta_{\rho_{i-1}^{*}}$ we have

$$
\begin{aligned}
v_{2}\left(X_{\rho_{i}^{*}}^{i}\right)=E_{X_{\rho_{i}^{*}}^{i}}\left[\int_{0}^{\tau^{(i+1) *} \wedge \sigma^{(i+1) *}} e^{-\alpha s} f\left(X_{s}^{i+1}\right) d s\right. \\
+\mathbf{1}_{\left\{\tau^{(i+1) *} \leq \sigma^{(i+1) *}\right\}} e^{-\alpha \tau^{(i+1) *}} M_{1}^{h} v_{1}\left(X_{\left.\tau^{(i+1) *}\right)}\right. \\
\left.+\mathbf{1}_{\left\{\sigma^{(i+1) *}<\tau^{(i+1) *}\right\}} e^{-\alpha \sigma^{(i+1) *}} M_{2}^{0} v_{2}\left(X_{\sigma^{(i+1) *}}\right)\right] .
\end{aligned}
$$

Iterating these equations and using the fact that, by Proposition $6.7, \rho_{n}^{*} \rightarrow \infty$, as $n \rightarrow \infty$ one can show that $\mathcal{J}^{V_{1}^{*}, V_{2}^{*}}(x)=v(x)$. Moreover, similarly as in [22] or [21], we can show that $V_{1}^{*}$ and $V_{2}^{*}$ form saddle-point strategies so that we have the existence of the value of the game within the class of shifted strategies. When we have other shifted strategies $V_{1}$ and $V_{2}$ then we easily show (using Theorem 3.1) that $\mathcal{J}^{V_{1}, V_{2}^{*}}(x) \leq$ $\mathcal{J}^{V_{1}^{*}, V_{2}^{*}}(x)=v(x) \leq \mathcal{J}^{V_{1}^{*}, V_{2}}(x)=v(x)$. We exploit here again Theorem 3.1, which therefore shows that for shifted strategies we have a sequence of stopping games.

7. Verification theorem and proof of the main result. In the previous sections 5 and 6 we have proved the existence of the value functions for shifted strategies under (A1) or under (A2), respectively. We now show that the value of the game is the same if we consider the most general (history-dependent) class of admissible impulse strategies. We first consider the case under the assumption (A1). Let $v$ be a solution to (4.1). For a given strategy $V_{2} \stackrel{\text { def }}{=}\left\{\sigma_{1}, \zeta_{1} ; \sigma_{2}, \zeta_{2} ; \ldots\right\}$ of the minimizer we shall define recursively (with a small abuse of notation) the strategy $\hat{V}_{1} \stackrel{\text { def }}{=}\left\{\hat{\tau}_{1}, \hat{\xi}_{1} ; \hat{\tau}_{2}, \hat{\xi}_{2} ; \ldots\right\}$ of the maximizer as follows:

Let $\rho_{0}^{V_{1}}=\rho_{0}^{V_{2}}=0$ and for $i=1,2, \ldots, \rho_{i}^{V_{2}}=\hat{\tau}_{i} \wedge \sigma_{i}$ and $\hat{\rho}_{i}^{V_{2}}=\rho_{i}^{V_{2}}+h\left(X_{\rho_{i}^{V_{2}}}^{i}, \eta_{i}\right)$, where $\eta_{i}=\hat{\xi}_{i}$ when $\hat{\tau}_{i} \leq \sigma_{i}$ or $\eta_{i}=\zeta_{i}$ otherwise, and where

$$
\hat{\tau}_{i}:=\inf \left\{s \geq \hat{\rho}_{i-1}^{V_{2}}: v\left(X_{s}^{i}\right)=M_{1}^{h} v\left(X_{s}^{i}\right)\right\}
$$

with $\hat{\xi}_{i}=\xi^{h}\left(X_{\hat{\tau}_{i}}^{i}\right)$, where $\xi^{h}$ is a selector of $M_{1}^{h}$ defined in (4.2). Similarly for a given $V_{1} \stackrel{\text { def }}{=}\left\{\tau_{1}, \xi_{1} ; \tau_{2}, \xi_{2} ; \ldots\right\}$ of the maximizer we shall define recursively the strategy $\hat{V}_{2} \stackrel{\text { def }}{=}\left\{\hat{\sigma}_{1}, \hat{\zeta}_{1} ; \hat{\sigma}_{2}, \hat{\zeta}_{2} ; \ldots\right\}$ of the minimizer as follows:

$$
\hat{\sigma}_{i}:=\inf \left\{s \geq \hat{\rho}_{i-1}^{V_{1}}: v\left(X_{s}^{i}\right)=M_{1}^{h} v\left(X_{s}^{i}\right) \vee M_{2}^{h} v\left(X_{s}^{i}\right)\right\}
$$

with $\rho_{i}^{V_{1}}=\tau_{i} \wedge \hat{\sigma}_{i}$ and $\hat{\rho}_{i}^{V_{1}}=\rho_{i}^{V_{1}}+h\left(X_{\rho_{i}^{V_{1}}}^{i}, \eta_{i}\right)$, where $\eta_{i}=\hat{\xi}_{i}$ when $\hat{\tau}_{i} \leq \sigma_{i}$ or $\eta_{i}=\zeta_{i}$ and $\hat{\zeta}_{i}=\zeta^{h}\left(X_{\hat{\sigma}_{i}}^{i}\right)$, where $\zeta^{h}$ is a selector of $M_{2}^{h}$ defined in (4.3) and $\hat{\tau}_{i}>\sigma_{i}$.

LEMma 7.1. For a given strategy $V_{2}=\left\{\sigma_{1}, \zeta_{1} ; \sigma_{2}, \zeta_{2} ; \ldots\right\}$ of the minimizer and the strategy $\hat{V}_{1}$ of the maximizer with $\hat{\tau}_{i}$ defined in $(7.1)$ we have for $i=1,2, \ldots$ that

$$
m_{1}^{i}(t):=\int_{\hat{\rho}_{i}^{V_{2}}}^{\left(\hat{\rho}_{i}^{V_{2}}+t\right) \wedge \hat{\tau}_{i+1}} e^{-\alpha s} f\left(X_{s}^{i}\right) d s+e^{-\alpha\left(\left(\hat{\rho}_{i}^{V_{2}}+t\right) \wedge \hat{\tau}_{i+1}\right)} v\left(X_{\left(\hat{\rho}_{i}^{V_{2}}+t\right) \wedge \hat{\tau}_{i+1}}\right)
$$

is a $\mathcal{G}_{t}^{i}:=\mathcal{F}_{\left(\hat{\rho}_{i}^{V_{2}}+t\right)}$ submartingale. Similarly for a given strategy $V_{1}=\left\{\tau_{1}, \xi_{1} ; \tau_{2}, \xi_{2} ; \ldots\right\}$ 
of the maximizer and the strategy $\hat{V}_{2}$ of the minimizer with $\hat{\sigma}_{i}$ defined in (7.2) we have for $i=1,2, \ldots$ that

$$
m_{2}^{i}(t):=\int_{\hat{\rho}_{i}^{V_{1}}}^{\left(\hat{\rho}_{i}^{V_{1}}+t\right) \wedge \hat{\sigma}_{i+1}} e^{-\alpha s} f\left(X_{s}^{i}\right) d s+e^{-\alpha\left(\left(\hat{\rho}_{i}^{V_{1}}+t\right) \wedge \hat{\sigma}_{i+1}\right)} v\left(X_{\left(\hat{\rho}_{i}^{V_{1}}+t\right) \wedge \hat{\sigma}_{i+1}}\right)
$$

is a $\mathcal{G}_{t}^{i}:=\mathcal{F}_{\left(\hat{\rho}_{i}^{V_{1}}+t\right)}$ supermartingale.

Proof. We have to show that $E\left[m_{1}^{i}(t+r) \mid \mathcal{G}_{t}^{i}\right] \geq m_{1}^{i}(t)$ and $E\left[m_{2}^{i}(t+r) \mid \mathcal{G}_{t}^{i}\right] \leq$ $m_{2}^{i}(t)$ for $r \geq 0$. It follows in a standard way from the construction of the probability space given in section 2 and well as (3.4) and (3.5) of Theorem 3.1. In fact for $u \geq 0$, we have using (3.4)

$$
\begin{aligned}
& E\left[m_{1}^{i}(t+u) \mid \mathcal{G}_{t}^{i}\right] \\
& =E\left[\int_{\hat{\rho}_{i}^{V_{2}}}^{\left(\hat{\rho}_{i}^{V_{2}}+t+u\right) \wedge \hat{\tau}_{i+1}} e^{-\alpha s} f\left(X_{s}^{i}\right) d s+e^{-\alpha\left(\left(\hat{\rho}_{i}^{V_{2}}+t+u\right) \wedge \hat{\tau}_{i+1}\right)} v\left(X_{\left(\hat{\rho}_{i}^{V_{2}}+t+u\right) \wedge \hat{\tau}_{i+1}}\right) \mid \mathcal{G}_{t}^{i}\right] \\
& \geq \int_{\hat{\rho}_{i}^{V_{2}}}^{\left(\hat{\rho}_{i}^{V_{2}}+t\right) \wedge \hat{\tau}_{i+1}} e^{-\alpha s} f\left(X_{s}^{i}\right) d s+e^{-\alpha\left(\left(\hat{\rho}_{i}^{V_{2}}+t\right) \wedge \hat{\tau}_{i+1}\right)} v\left(X_{\left(\hat{\rho}_{i}^{V_{2}}+t\right) \wedge \hat{\tau}_{i+1}}\right) \equiv m_{1}^{i}(t)
\end{aligned}
$$

and the proof of (7.4) is similar using (3.5).

In the case under the assumption (A2) we consider solutions $v_{1}, v_{2}, v$ to the IsaacsBellman equation (4.6)-(4.8) and define

$$
\hat{\tau}_{1}:=\inf \left\{s \geq 0: v\left(X_{s}^{1}\right)=M_{1}^{0} v\left(X_{s}^{1}\right)\right\}
$$

and

$$
\hat{\sigma}_{1}:=\inf \left\{s \geq 0: v\left(X_{s}^{1}\right)=M_{1}^{0} v\left(X_{s}^{1}\right) \vee M_{2}^{0} v\left(X_{s}^{1}\right)\right\} .
$$

For a given strategy $V_{2}=\left\{\sigma_{1}, \zeta_{1} ; \sigma_{2}, \zeta_{2} ; \ldots\right\}$ of the minimizer let $\rho_{1}^{V_{2}}=\hat{\rho}_{1}^{V_{2}}:=\hat{\tau}_{1} \wedge \sigma_{1}$. For $i=2$ when $\rho_{1}^{V_{2}}=\hat{\tau}_{1}$,

$$
\hat{\tau}_{2}:=\inf \left\{s \geq \rho_{1}^{V_{2}}: v_{1}\left(X_{s}^{2}\right)=M_{1}^{0} v_{1}\left(X_{s}^{2}\right)\right\}
$$

while when $\rho_{1}^{V_{2}}=\sigma_{1}<\hat{\tau}_{1}$

$$
\hat{\tau}_{2}:=\inf \left\{s \geq \rho_{1}^{V_{2}}: v_{2}\left(X_{s}^{2}\right)=M_{1}^{h} v_{1}\left(X_{s}^{2}\right)\right\} .
$$

Furthermore for $i>2$, when $\hat{\rho}_{i-1}^{V_{2}}=\hat{\tau}_{i-1}+h\left(X_{\hat{\tau}_{i-1}}, \hat{\xi}_{i}\right)$ or $\hat{\rho}_{i-1}^{V^{2}}=\hat{\tau}_{i-1}$ depending on whether we have or don't have at time $\rho_{i-1}^{V_{2}}=\hat{\tau}_{i-1} \wedge \sigma_{i-1}$ a decision lag of $h$, we have

$$
\hat{\tau}_{i}:=\inf \left\{s \geq \hat{\rho}_{i-1}^{V_{2}}: v_{1}\left(X_{s}^{i}\right)=M_{1}^{0} v_{1}\left(X_{s}^{i}\right)\right\},
$$

while when $\rho_{i-1}^{V_{2}}=\sigma_{i-1}<\hat{\tau}_{i-1}$

$$
\hat{\tau}_{i}:=\inf \left\{s \geq \hat{\rho}_{i-1}^{V_{2}}: v_{2}\left(X_{s}^{i}\right)=M_{1}^{h} v_{1}\left(X_{s}^{i}\right)\right\}
$$

with $\hat{\rho}_{i-1}^{V_{2}}=\hat{\tau}_{i-1} \vee \sigma_{i-1}$ in this case.

Copyright $@$ by SIAM. Unauthorized reproduction of this article is prohibited. 
For a given strategy $V_{1}=\left\{\tau_{1}, \xi_{1} ; \tau_{2}, \xi_{2} ; \ldots\right\}$ of the maximizer let $\rho_{1}^{V_{1}}=\hat{\rho}_{1}^{V_{1}}:=$ $\tau_{1} \wedge \hat{\sigma}_{1}$. For $i=2$ when $\rho_{1}^{V_{1}}=\tau_{1}$,

$$
\hat{\sigma}_{2}:=\inf \left\{s \geq \rho_{1}^{V_{1}}: v_{1}\left(X_{s}^{2}\right)=M_{1}^{0} v_{1}\left(X_{s}^{2}\right) \vee M_{2}^{0} v_{2}\left(X_{s}^{2}\right)\right\},
$$

while when $\rho_{1}^{V_{1}}=\hat{\sigma}_{1}<\tau_{1}$

$$
\hat{\sigma}_{2}:=\inf \left\{s \geq \rho_{1}^{V_{1}}: v_{2}\left(X_{s}^{2}\right)=M_{1}^{h} v_{1}\left(X_{s}^{2}\right) \vee M_{2}^{0} v_{2}\left(X_{s}^{2}\right)\right\} .
$$

Furthermore for $i>2$, when $\hat{\rho}_{i-1}^{V_{1}}=\tau_{i-1}+h\left(X_{\tau_{i-1}}^{i-1}, \xi_{i}\right)$, or $\hat{\rho}_{i-1}^{V^{1}}=\tau_{i-1}$ depending on whether we have or don't have at time $\rho_{i-1}^{V_{1}}=\hat{\tau}_{i-1} \wedge \sigma_{i-1}$ a decision lag of $h$, we have

$$
\hat{\sigma}_{i}:=\inf \left\{s \geq \hat{\rho}_{i-1}^{V_{1}}: v_{1}\left(X_{s}^{i}\right)=M_{1}^{0} v_{1}\left(X_{s}^{i}\right) \vee M_{2}^{0} v_{2}\left(X_{s}^{i}\right)\right\},
$$

while when $\hat{\rho}_{i-1}^{V_{1}}=\hat{\sigma}_{i-1}<\tau_{i-1}$

$$
\hat{\sigma}_{i}:=\inf \left\{s \geq \hat{\rho}_{i-1}^{V_{1}}: v_{2}\left(X_{s}^{i}\right)=M_{1}^{h} v_{1}\left(X_{s}^{i}\right) \vee M_{2}^{0} v_{2}\left(X_{s}^{i}\right)\right\} .
$$

with $\hat{\rho}_{i-1}^{V_{1}}=\rho_{i-1}^{V_{1}}$.

By analogy to Lemma 7.1 we obtain the following.

Lemma 7.2. For a given strategy $V_{2}=\left\{\sigma_{1}, \zeta_{1} ; \sigma_{2}, \zeta_{2} ; \ldots\right\}$ of the minimizer and the strategy $\hat{V}_{1}=\left\{\hat{\tau}_{1}, \hat{\xi}_{1} ; \hat{\tau}_{2}, \hat{\xi}_{2} ; \ldots\right\}$ of the maximizer we have for $i=1,2, \ldots$ that whenever $\rho_{i}^{V_{2}}=\hat{\tau}_{i}$ with $\hat{\tau}_{i}$ defined in (7.6) and (7.8) then

$$
m_{1}^{i}(t):=\int_{\hat{\rho}_{i}^{V_{2}}}^{\left(\hat{\rho}_{i}^{V_{2}}+t\right) \wedge \hat{\tau}_{i+1}} e^{-\alpha s} f\left(X_{s}^{i}\right) d s+e^{-\alpha\left(\left(\hat{\rho}_{i}^{V_{2}}+t\right) \wedge \hat{\tau}_{i+1}\right)} v_{1}\left(X_{\left(\hat{\rho}_{i}^{V_{2}}+t\right) \wedge \hat{\tau}_{i+1}}\right)
$$

is a $\mathcal{G}_{t}^{i}:=\mathcal{F}_{\left(\hat{\rho}_{i}^{V_{2}}+t\right)}$ submartingale, while whenever $\hat{\rho}_{i}^{V_{2}}=\sigma_{i}<\hat{\tau}_{i}$ with $\hat{\tau}_{i}$ defined in (7.9) then

$$
m_{1}^{i}(t):=\int_{\hat{\rho}_{i}^{V_{2}}}^{\left(\tilde{\rho}_{i}^{V_{2}}+t\right) \wedge \hat{\tau}_{i+1}} e^{-\alpha s} f\left(X_{s}^{i}\right) d s+e^{-\alpha\left(\left(\tilde{\rho}_{i}^{V_{2}}+t\right) \wedge \hat{\tau}_{i+1}\right)} v_{2}\left(X_{\left(\tilde{\rho}_{i}^{V_{2}}+t\right) \wedge \hat{\tau}_{i+1}}\right)
$$

$\mathcal{G}_{t}^{i}:=\mathcal{F}_{\left(\hat{\rho}_{i}^{V_{2}}+t\right)}$ submartingale. For a given strategy $V_{1}=\left\{\tau_{1}, \xi_{1} ; \tau_{2}, \xi_{2} ; \ldots\right\}$ of the maximizer and the strategy $\hat{V}_{2}=\left\{\hat{\sigma}_{1}, \hat{\zeta}_{1} ; \hat{\sigma}_{2}, \hat{\zeta}_{2} ; \ldots\right\}$ whenever $\rho_{i}^{V_{1}}=\tau_{i}$ with $\hat{\sigma}_{i}$ defined in (7.7) and (7.12) we have that

$$
m_{2}^{i}(t):=\int_{\hat{\rho}_{i}^{V_{1}}}^{\left(\hat{\rho}_{i}^{V_{1}}+t\right) \wedge \sigma_{i+1}} e^{-\alpha s} f\left(X_{s}^{i}\right) d s+e^{-\alpha\left(\left(\hat{\rho}_{i}^{V_{1}}+t\right) \wedge \sigma_{i+1}\right)} v_{1}\left(X_{\left(\hat{\rho}_{i}^{V_{1}}+t\right) \wedge \sigma_{i+1}}\right)
$$

is a $\mathcal{G}_{t}^{i}=\mathcal{F}_{\left(\hat{\rho}_{i}^{V_{1}}+t\right)}$ supermartingale, while whenever $\rho_{i}=\hat{\sigma}_{i}<\tau_{i}$ with $\hat{\sigma}_{i}$ defined in (7.13) we have that

$$
m_{2}^{i}(t):=\int_{\hat{\rho}_{i}^{V_{1}}}^{\left(\hat{\rho}_{i}^{V_{1}}+t\right) \wedge \hat{\sigma}_{i+1}} e^{-\alpha s} f\left(X_{s}^{i}\right) d s+e^{-\alpha\left(\left(\hat{\rho}_{i}^{V_{1}}+t\right) \wedge \hat{\sigma}_{i+1}\right)} v_{2}\left(X_{\left(\hat{\rho}_{i}^{V_{1}}+t\right) \wedge \hat{\sigma}_{i+1}}\right)
$$

is a $\mathcal{G}_{t}^{i}=\mathcal{F}_{\left(\hat{\rho}_{i}^{V_{1}}+t\right)}$ supermartingale.

Copyright (c) by SIAM. Unauthorized reproduction of this article is prohibited. 
We shall now formulate and prove the following proposition, which completes the proof of Theorem 4.2 under all general admissible strategies. In this case the following holds.

Proposition 7.3. Under (A1) or under (A2) there are saddle-point strategies $\hat{V}_{1}=\left\{\hat{\tau}_{1}, \hat{\xi}_{1} ; \hat{\tau}_{2}, \hat{\xi}_{2} ; \ldots\right\}$ and $\hat{V}_{2}=\left\{\hat{\sigma}_{1}, \hat{\zeta}_{1} ; \hat{\sigma}_{2}, \hat{\zeta}_{2} ; \ldots\right\}$ for the functional (2.1). These strategies are of the following form:

- Under (A1), $\hat{\tau}_{i}$ and $\hat{\sigma}_{i}$ are defined in (7.1) and (7.2), respectively, and $\hat{\xi}_{i}=$ $\xi^{h}\left(X_{\hat{\tau}_{i}}^{i}\right), \hat{\zeta}_{i}=\zeta^{h}\left(X_{\hat{\sigma}_{i}}^{i}\right)$, where $\xi^{h}$ and $\zeta^{h}$ are selectors for $M_{1}^{h}$ and $M_{2}^{h}$ defined in (4.2) and (4.3), respectively.

- Under (A2), $\hat{\tau}_{1}$ and $\hat{\sigma}_{1}$ are defined in (7.6) and (7.7), respectively, $\hat{\xi}_{1}=$ $\xi^{0}\left(X_{\hat{\tau}_{1}}^{1}\right), \hat{\zeta}_{1}=\zeta^{h}\left(X_{\hat{\sigma}_{1}}^{1}\right)$, while for $i \geq 2$ when $\hat{\tau}_{i-1} \leq \hat{\sigma}_{i-1}$ stopping times $\hat{\tau}_{i}$ and $\hat{\sigma}_{i}$ are defined in (7.8) and (7.12), respectively, and $\hat{\xi}_{i}=\xi^{0}\left(X_{\hat{\tau}_{i}}^{i}\right)$, $\hat{\zeta}_{i}=\zeta^{0}\left(X_{\hat{\sigma}_{i}}^{i}\right)$; when $\hat{\sigma}_{i-1}<\hat{\tau}_{i-1}$ stopping times $\hat{\tau}_{i}$ and $\hat{\sigma}_{i}$ are defined in $(7.9)$ and (7.13), respectively, and $\hat{\xi}_{i}=\xi^{h}\left(X_{\hat{\tau}_{i}}^{i}\right), \hat{\zeta}_{i}=\zeta^{0}\left(X_{\hat{\sigma}_{i}}^{i}\right)$, where $\xi^{0}, \zeta^{0}$ are selectors for $M_{1}^{0}$ and $M_{2}^{0}$ defined in (4.2) and (4.3), respectively, with $h \equiv 0$.

Finally, the function $v$ defined as a solution to (4.1) under (A1) or defined as a solution to (4.8) under (A2) is the value of the game.

Proof. Notice first that for stopping times $\hat{V}_{1}$ and $\hat{V}_{2}$ we have function $v(x)=$ $\mathcal{J}\left(\hat{V}_{1}, \hat{V}_{2}\right)$ and clearly $\hat{V}_{1}$ and $\hat{V}_{2}$ are shifted strategies. Now, under (A1), in the case of an arbitrary strategy $V_{2}=\left\{\sigma_{1}, \zeta_{1} ; \sigma_{2}, \zeta_{2} ; \ldots\right\}$ when $\rho_{i}^{V_{2}}=\hat{\tau}_{i}$ by letting for simplicity $\bar{h}_{i}:=h\left(X_{\rho_{i}}^{i-1}, \hat{\xi}_{i}\right)$ and using $(7.3)$ and taking into account that $M_{1}^{h} v(x) \leq$ $v(x) \leq M_{1}^{h} v(x) \wedge M_{2}^{h} v(x)$ we have

$$
\begin{aligned}
& v\left(X_{\rho_{i}^{V_{2}}}^{i-1}\right)= M_{1}^{h} v\left(X_{\rho_{i}^{V_{2}}}^{i-1}\right) \\
&= c\left(X_{\rho_{i}^{V_{2}}}^{i-1}, \hat{\xi}_{i}\right)+E\left[\int_{\rho_{i}^{V_{2}}}^{\rho_{i}^{V_{2}}+\bar{h}_{i}} e^{-\alpha s} f\left(X_{s}^{i}\right) d s+e^{-\alpha \rho_{i}^{V_{2}}+\bar{h}_{i}} v\left(X_{\rho_{i}^{V_{2}}+\bar{h}_{i}}\right) \mid \mathcal{F}_{\rho_{i}^{V_{2}}}\right] \\
& \leq c\left(X_{\rho_{i}^{V_{2}}}^{i}, \hat{\xi}_{i}\right)+E\left[\int_{\rho_{i}^{V_{2}}}^{\rho_{i}^{V_{2}}+\bar{h}_{i}} e^{-\alpha s} f\left(X_{s}^{i}\right) d s+E\left[\int_{\hat{\rho}_{i}^{V_{2}}}^{\sigma_{i+1} \wedge \hat{\tau}_{i+1}} e^{-\alpha s} f\left(X_{s}^{i}\right) d s\right.\right. \\
&\left.\left.+e^{-\alpha\left(\sigma_{i+1} \wedge \hat{\tau}_{i+1}\right)} v\left(X_{\sigma_{i+1} \wedge \hat{\tau}_{i+1}}\right) \mid \mathcal{F}_{\hat{\rho}_{i}}\right] \mid \mathcal{F}_{\rho_{i}^{V_{2}}}\right] \\
& \leq c\left(X_{\rho_{i}^{V_{2}}}^{i-1}, \hat{\xi}_{i}\right)+E\left[\int_{\rho_{i}^{V_{2}}}^{\hat{\tau}_{i+1} \wedge \sigma_{i+1}} e^{-\alpha s} f\left(X_{s}^{i}\right) d s+\mathbf{1}_{\left\{\hat{\tau}_{i+1} \leq \sigma_{i+1}\right\}} e^{-\alpha \hat{\tau}_{i+1}} M_{1}^{h} v\left(X_{\hat{\tau}_{i+1}}\right)\right. \\
&\left.+\mathbf{1}_{\left\{\sigma_{i+1}<\hat{\tau}_{i+1}\right\}} e^{-\alpha \sigma_{i+1}}\left(M_{1}^{h} v\left(X_{\sigma_{i+1}}^{i}\right) \vee M_{2}^{h} v\left(X_{\sigma_{i+1}}^{i}\right)\right) \mid \mathcal{F}_{\rho_{i}^{V_{2}}}\right] .
\end{aligned}
$$

In the case when $\rho_{i}^{V_{2}}=\sigma_{i}<\hat{\tau}_{i}$, this time with $\bar{h}_{i}:=h\left(X_{\rho_{i}^{V_{2}}}^{i-1}, \zeta_{i}\right)$, we have

$$
\begin{aligned}
& v\left(X_{\rho_{i}^{V_{2}}}^{i-1}\right) \leq M_{2}^{h} v\left(X_{\rho_{i}^{V_{2}}}^{i-1}\right) \\
&=d\left(X_{\rho_{i}^{V_{2}}}^{i-1}, \zeta_{i}\right)+E\left[\int_{\rho_{i}^{V_{2}}}^{\hat{\rho}_{i}^{V_{2}}+\bar{h}_{i}} e^{-\alpha s} f\left(X_{s}^{i}\right) d s+e^{-\alpha \rho_{i}^{V_{2}}+\bar{h}_{i}} v\left(X_{\rho_{i}^{V_{2}}+\bar{h}_{i}}\right) \mid \mathcal{F}_{\rho_{i}^{V_{2}}}\right] \\
& \leq d\left(X_{\rho_{i}^{V_{2}}}^{i}, \zeta_{i}\right)+E\left[\int_{\rho_{i}^{V_{2}}}^{\rho_{i}^{V_{2}}+\bar{h}_{i}} e^{-\alpha s} f\left(X_{s}^{i}\right) d s+E\left[\int_{\hat{\rho}_{i}^{V_{2}}}^{\sigma_{i+1} \wedge \hat{\tau}_{i+1}} e^{-\alpha s} f\left(X_{s}^{i}\right) d s\right.\right. \\
&\left.\left.+e^{-\alpha\left(\sigma_{i+1} \wedge \hat{\tau}_{i+1}\right)} v\left(X_{\sigma_{i+1} \wedge \hat{\tau}_{i+1}}\right) \mid \mathcal{F}_{\hat{\rho}_{i}^{V_{2}}}\right] \mid \mathcal{F}_{\rho_{i}^{V_{2}}}\right]
\end{aligned}
$$

Copyright (c) by SIAM. Unauthorized reproduction of this article is prohibited. 


$$
\begin{aligned}
\leq d\left(X_{\rho_{i}}^{i-1}, \zeta_{i}\right)+E & {\left[\int_{\rho_{i}^{V_{2}}}^{\hat{\tau}_{i+1} \wedge \sigma_{i+1}} e^{-\alpha s} f\left(X_{s}^{i}\right) d s+\mathbf{1}_{\left\{\hat{\tau}_{i+1} \leq \sigma_{i+1}\right\}} e^{-\alpha \hat{\tau}_{i+1}} M_{1}^{h} v\left(X_{\hat{\tau}_{i+1}}\right)\right.} \\
& \left.+\mathbf{1}_{\left\{\sigma_{i+1}<\hat{\tau}_{i+1}\right\}} e^{-\alpha \sigma_{i+1}}\left(M_{1}^{h} v\left(X_{\sigma_{i+1}}^{i}\right) \vee M_{2}^{h} v\left(X_{\sigma_{i+1}}^{i}\right)\right) \mid \mathcal{F}_{\rho_{i}}\right]
\end{aligned}
$$

so that by induction we obtain that $v(x) \leq \mathcal{J}\left(\hat{V}_{1}, V_{2}\right)$. Similarly, using (7.4), for an arbitrary strategy $V_{1}=\left\{\tau_{1}, \xi_{1} ; \tau_{2}, \xi_{2} ; \ldots\right\}$ whenever $\rho_{i}^{V_{2}}=\tau_{i}$ by letting for simplicity $\bar{h}_{i}:=h\left(X_{\rho_{i}^{V_{1}}}^{i-1}, \xi_{i}\right)$ we have

$$
\begin{aligned}
& v\left(X_{\rho_{i}^{V_{1}}}^{i-1}\right) \geq M_{1}^{h} v\left(X_{\rho_{i}^{V_{1}}}^{i-1}\right) \\
& \geq c\left(X_{\rho_{i}^{V_{1}}}^{i-1}, \xi_{i}\right)+E\left[\int_{\rho_{i}^{V_{1}}}^{\rho_{i}^{V_{1}}+\bar{h}_{i}} e^{-\alpha s} f\left(X_{s}^{i}\right) d s+e^{-\alpha \rho_{i}^{V_{1}}+\bar{h}_{i}} v\left(X_{\rho_{i}^{V_{1}}+\bar{h}_{i}}\right) \mid \mathcal{F}_{\rho_{i}^{V_{1}}}\right] \\
& \geq c\left(X_{\rho_{i}^{V_{1}}}^{i}, \xi_{i}\right)+E\left[\int_{\rho_{i}^{V_{1}}}^{\rho_{i}^{V_{1}}+\bar{h}_{i}} e^{-\alpha s} f\left(X_{s}^{i}\right) d s+E\left[\int_{\hat{\rho}_{i}^{V_{2}}}^{\tau_{i+1} \wedge \hat{\sigma}_{i+1}} e^{-\alpha s} f\left(X_{s}^{i}\right) d s\right.\right. \\
&\left.\left.+e^{-\alpha\left(\tau_{i+1} \wedge \hat{\sigma}_{i+1}\right)} v\left(X_{\tau_{i+1} \wedge \hat{\sigma}_{i+1}}\right) \mid \mathcal{F}_{\hat{\rho}_{i}^{V_{2}}}\right] \mid \mathcal{F}_{\rho_{i}^{V_{2}}}\right] \\
& \leq c\left(X_{\rho_{i}^{V_{2}}}^{i-1}, \xi_{i}\right)+E\left[\int_{\rho_{i}^{V_{1}}}^{\tau_{i+1} \wedge \hat{\sigma}_{i+1}} e^{-\alpha s} f\left(X_{s}^{i}\right) d s+\mathbf{1}_{\left\{\tau_{i+1} \leq \hat{\sigma}_{i+1}\right\}} e^{-\alpha \tau_{i+1}} M_{1}^{h} v\left(X_{\tau_{i+1}}\right)\right. \\
&\left.+\mathbf{1}_{\left\{\hat{\sigma}_{i+1}<\tau_{i+1}\right\}} e^{-\alpha \hat{\sigma}_{i+1}}\left(M_{1}^{h} v\left(X_{\hat{\sigma}_{i+1}}^{i}\right) \vee M_{2}^{h} v\left(X_{\hat{\sigma}_{i+1}}^{i}\right)\right) \mid \mathcal{F}_{\rho_{i}^{V_{1}}}\right]
\end{aligned}
$$

and similarly whenever $\rho_{i}^{V_{2}}=\hat{\sigma}_{i}$ from which in turn we obtain that $v(x) \geq \mathcal{J}\left(V_{1}, \hat{V}_{2}\right)$. The case under (A2) can be proved in a similar way. The main technical difference is that we have to use the system of Bellman equations (4.6) and (4.7) and therefore by Lemma 7.2 we have two submartingales (7.16) and (7.17) depending on the value of $\rho_{i}^{V_{2}}$ and two supermartingales (7.18) and (7.19) depending on the value of $\rho_{i}^{V_{1}}$. By analogy to the proof of the case under (A1) we see that we have two times more cases to consider, but the structure of the proof is similar and is correspondingly based on Lemma 7.2. We shall therefore have more technicalities but it does not change the main idea of the proof. Therefore it is left to the reader.

8. Conclusion and future directions. In this paper a zero-sum Markov game on general state spaces with impulses and infinite time horison discounted payoff is considered under very weak assumptions and weak Feller conditions on the state process. The existence of value and saddle-point (optimal) strategies are proved. One of the key contributions is our analysis of this problem based on shifted strategies (see section 2), thereby proving that the original game can be practically restricted to a sequence of Dynkin's stopping games without affecting the optimalty of the saddle-point equilibria. We also prove the necessity of our assumptions by providing a counterexample (see Example 1) which shows that the game with impulses makes sense only when there are some kind of assumptions which enable us to restrict an infinite number of immediate impulses. A point to note is that we have used purely probabilistic techniques in our proofs which are different from the previous proof methodology used in the existing literature. As a consequence, we establish an interesting property of the underlying Feller-Markov process and the impossibility of an infinite number of impulses in finite time under saddle-point strategies, which is crucial for the verification result of the corresponding Isaacs-Bellman equations. Thus, in this paper, we 
completely solve the open problem similar to that in [9] under a very general set of assumptions and establish the corresponding saddle-point (optimal) strategies, thereby fully generalizing [9]. The study of this problem was motivated by (among others) Israeli swing options with multiple exercises (see [12]). Therefore our general result can be further used to price this kind of Israeli option assuming Markovian structure of asset prices, e.g., log-Levy prices, which may be a subject of further finance-oriented research. Another possible extension of our paper is to consider average cost per unit time payoff for this kind of game generalizing the paper [20] to the game-theoretic scenario. Further possible extensions to risk-sensitive payoffs and/or to nonzero-sum games with impulses seem to be harder at the moment and shall require a number of preliminary results first.

Acknowledgments. The authors would like to most sincerely thank the reviewer for providing extremely insightful technical comments which helped them immensely in substantially improving the technical depth and breadth of this manuscript, leading to several major changes. Finally, the authors would also like to most sincerely thank the Corresponding Editor Prof. Dr. Francois Dufour and the associate editor for providing substantial time upon the authors' request to revise this manuscript in the light of the deep review comments made by the reviewer.

\section{REFERENCES}

[1] P. Azimzadeh (2019), A zero-sum stochastic differential game with impulses, precommitment, and unrestricted cost functions, Appl. Math. Optim., 79, pp. 483-514.

[2] A. Bensoussan And A. Friedman (1974), Nonlinear variational inequalities and differential games with stopping times, J. Funct. Anal., 16, pp. 305-352.

[3] J. M. Bismut (1977), Sur une probleme de Dynkin, Z. Wahr. Verw. Gebiete, 39, pp. 31-53.

[4] A. Cosso (2013), Stochastic differential games involving impulse controls and double-obstacle quasi-variational inequalities, SIAM J. Control Optim., 51, pp. 2102-2131.

[5] J. CVITANic AND I. Karatzas (1996), Backward stochastic differential equations with reflection and Dynkin games, Ann. Probab., 26, pp. 2024-2056.

[6] E. B. Dynkin (1965), Markov Processes, Springer, Berlin.

[7] E. B. Dynkin (1969), The game variant of the optimal stopping problem, Dokl. Akad. Nauk USSR, 185 , pp. 16-19.

[8] E. Ekström And G. Peskir (2000), Optimal stopping games for Markov processes, SIAM J. Control Optim., 47, pp. 684-702.

[9] B. El Asri And S. Mazid (2018), Zero-Sum Stochastic Differential Game in Finite Horizon Involving Impulse Controls, Appl. Math. Optim., doi:10.1007/S00245-018-9529-2.

[10] S. HamadÉne (2006), Mixed zero-sum stochastic differential game and American game options, SIAM J. Control Optim., 45, pp. 496-518.

[11] S. HAMAdÉNe AND J. ZHANG (2010), The continuous time nonzero-sum Dynkin game problem and application in game option, SIAM J. Control Optim., 48, pp. 3659-3669.

[12] Y. Iron AND Y. Kifer (2011), Hedging of swing game options in continuous time, Stochastics, 83, pp. $365-404$.

[13] Y. Kifer (2000), Game options, Finance Stoch., 4, pp. 443-463.

[14] N. V. KRYlov (1970), The problem with two free boundaries for an equation and optimal stopping of Markov processes, Dokl. Akad. Nauk USSR, 194, pp. 1263-1265.

[15] N. V. KRYlov (1971), Control of Markov processes and the spaces, W. Math. USSR Izv., 5, pp. 233-266.

[16] R. LARAKi AND E. Solan (2005), The value of zero-sum stopping games in continuous time, SIAM J. Control Optim., 43, pp. 1913-1922.

[17] J. P. Lepeltier and M. A. Maingueneau (1984), Le jeu de Dynkin en théorie générale sans l'hypothése de Mokobodski, Stochastics, 13, pp. 25-44.

[18] J. P. Lepeltier and B. Marchal (1984), Theorie generale du control impulsionnel Markovien, SIAM J. Control Optim., 22, pp. 645-665.

[19] J. Palczewski And Ł. Stettner (2010), Finite horizon optimal stopping of timediscontinuous functionals with applications to impulse control with delay, SIAM J. Control Optim., 48, pp. 4874-4909. 
[20] J. Palczewski and Ł. Stettner (2017), Impulse control maximising average cost per unit time: A non-uniformly ergodic case, SIAM J. Control Optim., 55, pp. 936-960.

[21] M. Robin (1978), Controle impulsionnel des processus de Markov, Thèse détat, University of Paris IX, https://tel.archives-ouvertes.fr/tel-00735779.

[22] E. Stettner (1982), Zero-sum Markov games with stopping and impulsive strategies, Appl. Math. Optim., 9, pp. 1-24.

[23] Ł. Stettner (2011), Penalty method for finite horizon stopping problems, SIAM J. Control Optim., 49, pp. 1078-1999.

[24] E. Stettner (1983), On impulsive control with long run average cost criterion, Studia Math., 76, pp. 279-298.

[25] Ł. Stettner (1984), On closedness of general zero-sum stopping game, Bull. Polish Acad. Sci. Math., 32, pp. 351-461.

[26] N. Touzi And N. Vielle (2002), Continuous-time Dynkin games with mixed strategies, SIAM J. Control Optim., 4, pp. 1073-1088.

Copyright (c) by SIAM. Unauthorized reproduction of this article is prohibited. 